\title{
Lattice Boltzmann model with self-tuning equation of state for multiphase flows
}

\author{
Rongzong Huang, ${ }^{1,2, *}$ Huiying $\mathrm{Wu}^{1, \dagger}$ and Nikolaus A. Adams ${ }^{2}, \ddagger$ \\ ${ }^{1}$ School of Mechanical Engineering, Shanghai Jiao Tong University, 200240 Shanghai, China \\ ${ }^{2}$ Institute of Aerodynamics and Fluid Mechanics, \\ Technical University of Munich, 85748 Garching, Germany
}

(Dated: September 10, 2018)

\begin{abstract}
A novel lattice Boltzmann (LB) model for multiphase flows is developed that complies with the thermodynamic foundations of kinetic theory. By directly devising the collision term for LB equation at the discrete level, a self-tuning equation of state is achieved, which can be interpreted as the incorporation of short-range molecular interaction. A pairwise interaction force is introduced to mimic the long-range molecular interaction, which is responsible for interfacial dynamics. The derived pressure tensor is naturally consistent with thermodynamic theory, and surface tension and interface thickness can be independently prescribed.
\end{abstract}

PACS numbers: 47.11.-j, 47.55.-t, 05.70.Ce, 05.20.Dd

The lattice Boltzmann (LB) method firstly introduced in 1988 [1] uses a set of distribution functions with discrete velocities to depict the complex fluid flows. Due to its kinetic nature, the LB method shows potential for considering microscopic and mesoscopic interactions. It is therefore believed that this method is particularly suitable for multiphase flows, which are complex at the macroscopic level but are much simpler from the microscopic viewpoint. The applications of LB method in multiphase flows emerged in the early 1990s [2] and have significantly increased in the past decade [3].

Although various LB models for multiphase flows exist [4-6], criticisms have been raised for a long time [7, 8]. In the pseudopotential LB model [4, 9], a pairwise interaction force is used to mimic the microscopic interaction, which can recover nonideal-gas effects and interfacial dynamics at the same time. However, such simultaneous recoveries make this model suffer from thermodynamic inconsistency, though significant progress has been made in approximating the coexistence densities close to the thermodynamic results [10-12]. In the free-energy LB model $[5,13]$, the thermodynamically consistent pressure tensor is directly incorporated to produce the dynamics of multiphase flows. Thus, the annoying evaluations of (highorder) derivatives are unavoidable, though improvements have been made to remedy the violation of Galilean invariance in this model [14-17]. Different from the pseudopotential and free-energy models, the multiphase LB model has also been developed from kinetic theory via systematic discretization procedures $[6,8]$. Complicated equivalent force terms exist in this model and severe numerical instability is encountered. Improved models were formulated $[18,19]$ at the price of sacrificing the underlying physics and computational simplicity.

In this work, we develop a novel LB model for multiphase flows complying with the thermodynamic foundations of kinetic theory analyzed by $\mathrm{He}$ and Doolen [8]. The underlying molecular interaction responsible for multiphase flows is divided into the short-range and long-range parts, which are incorporated by constructing an LB model with self-tuning equation of state (EOS) and introducing a pairwise interaction force, respectively. The present LB model has the advantages of the popular pseudopotential and free-energy LB models and is free of the aforementioned drawbacks.

With the presence of a discrete force term $F_{v, i}$, the LB equation for the density distribution function $f_{i}$ can be generally expressed as [20]

$$
f_{i}\left(\mathbf{x}+\mathbf{e}_{i} \delta_{t}, t+\delta_{t}\right)=f_{i}+\delta_{t} F_{v, i}-\Lambda_{i k}\left(f_{k}-f_{k}^{\mathrm{eq}}+\frac{\delta_{t}}{2} F_{v, k}\right),
$$

where $\mathbf{e}_{i}$ is the discrete velocity, $\Lambda_{i k}$ is the collision matrix in discrete velocity space, and the right-hand side (RHS), termed the collision process, is computed at position $\mathbf{x}$ and time $t$. Owing to the explicit physical significance of the moments of distribution function, it is more convenient to construct the collision term in moment space than in discrete velocity space. Orthogonal moments without weights are adopted [21], and the RHS of Eq. (1) is transformed into moment space

$$
\overline{\mathbf{m}}=\mathbf{m}+\delta_{t} \mathbf{F}_{m}-\mathbf{S}\left(\mathbf{m}-\mathbf{m}^{\mathrm{eq}}+\frac{\delta_{t}}{2} \mathbf{F}_{m}\right),
$$

where $\mathbf{m}=\mathbf{M}\left(f_{i}\right)^{\mathrm{T}}$ is the rescaled moment with $\mathbf{M}$ being the dimensionless transformation matrix [21], and $\overline{\mathbf{m}}$ denotes the post-collision moment. For the sake of simplicity, the two-dimensional nine-velocity (D2Q9) lattice is considered here [22], and the extension to threedimensional lattice is straightforward though tedious. The equilibrium moment function $\mathbf{m}^{\mathrm{eq}}=\mathbf{M}\left(f_{i}^{\mathrm{eq}}\right)^{\mathrm{T}}$ is devised as

$$
\begin{aligned}
\mathbf{m}^{\mathrm{eq}}=\left[\rho, 2 \alpha_{1} \rho\right. & +2 \beta_{1} \eta+3 \rho|\hat{\mathbf{u}}|^{2}, \alpha_{2} \rho+\beta_{2} \eta-3 \rho \times \\
|\hat{\mathbf{u}}|^{2}+ & 9 \rho \hat{u}_{x}^{2} \hat{u}_{y}^{2}, \rho \hat{u}_{x},-\rho \hat{u}_{x}+3 \rho \hat{u}_{x} \hat{u}_{y}^{2}, \rho \hat{u}_{y}, \\
& \left.-\rho \hat{u}_{y}+3 \rho \hat{u}_{y} \hat{u}_{x}^{2}, \rho\left(\hat{u}_{x}^{2}-\hat{u}_{y}^{2}\right), \rho \hat{u}_{x} \hat{u}_{y}\right]^{\mathrm{T}},
\end{aligned}
$$

where $\hat{\mathbf{u}}=\mathbf{u} / c$ with lattice speed $c=\delta_{x} / \delta_{t}, \eta$ is introduced to achieve the self-tuning EOS, $\alpha_{1,2}$ and $\beta_{1,2}$ 
are coefficients that will be determined later. The corresponding discrete force term in moment space $\mathbf{F}_{m}=$ $\mathbf{M}\left(F_{v, i}\right)^{\mathrm{T}}$ is set as follows

$$
\begin{array}{r}
\mathbf{F}_{m}=\left\{0,6 \hat{\mathbf{F}} \cdot \hat{\mathbf{u}},-6 \hat{\mathbf{F}} \cdot \hat{\mathbf{u}}+9[\hat{\mathbf{F}} \hat{\mathbf{u}} \hat{\mathbf{u}} \hat{\mathbf{u}}]_{x x y y}, \hat{F}_{x},\right. \\
-\hat{F}_{x}+3[\hat{\mathbf{F}} \hat{\mathbf{u}} \hat{\mathbf{u}}]_{x y y}, \hat{F}_{y},-\hat{F}_{y}+3[\hat{\mathbf{F}} \hat{\mathbf{u}} \hat{\mathbf{u}}]_{x x y}, \\
\left.2\left(\hat{F}_{x} \hat{u}_{x}-\hat{F}_{y} \hat{u}_{y}\right), \hat{F}_{x} \hat{u}_{y}+\hat{F}_{y} \hat{u}_{x}\right\}^{\mathrm{T}},
\end{array}
$$

where $\hat{\mathbf{F}}=\mathbf{F} / c,[\bullet]$ denotes permutation and the subscripts denote tensor indices. In Eqs. (3) and (4), the high-order terms of velocity correspond to the third- and fourth-order Hermite terms in $f_{i}^{\text {eq }}$ and $F_{v, i}$, whose effects will be discussed later. The macroscopic density $\rho$ and velocity $\mathbf{u}$ are defined as

$$
\rho=\sum_{i} f_{i}, \quad \rho \mathbf{u}=\sum_{i} \mathbf{e}_{i} f_{i}+\frac{\delta_{t}}{2} \mathbf{F} .
$$

Once the equilibrium distribution function in LB equation is changed to achieve self-tuning EOS, it has been recognized previously that Newtonian viscous stress cannot be recovered correctly, and Galilean invariance will be lost [13, 23]. From the Enskog equation for dense gases in kinetic theory, we note that an extra velocity-dependent term emerges in the collision term $[7,8,24]$. Inspired by this fact, some velocity-dependent non-diagonal elements are introduced in the collision matrix $\mathbf{S}$

$$
\mathbf{S}=\left[\begin{array}{ccccccccc}
s_{0} & 0 & 0 & 0 & 0 & 0 & 0 & 0 & 0 \\
0 & s_{e} & k s_{\varepsilon} \omega_{e} & 0 & h \hat{u}_{x} s_{q} \omega_{e} & 0 & h \hat{u}_{y} s_{q} \omega_{e} & 0 & 0 \\
0 & 0 & s_{\varepsilon} & 0 & 0 & 0 & 0 & 0 & 0 \\
0 & 0 & 0 & s_{j} & 0 & 0 & 0 & 0 & 0 \\
0 & 0 & 0 & 0 & s_{q} & 0 & 0 & 0 & 0 \\
0 & 0 & 0 & 0 & 0 & s_{j} & 0 & 0 & 0 \\
0 & 0 & 0 & 0 & 0 & 0 & s_{q} & 0 & 0 \\
0 & 0 & 0 & 0 & 2 b \hat{u}_{x} s_{q} \omega_{p} & 0 & -2 b \hat{u}_{y} s_{q} \omega_{p} & s_{p} & 0 \\
0 & 0 & 0 & 0 & b \hat{u}_{y} s_{q} \omega_{p} & 0 & b \hat{u}_{x} s_{q} \omega_{p} & 0 & s_{p}
\end{array}\right],
$$

where $\omega_{e, p}=s_{e, p} / 2-1$, and $k, h$ and $b$ are coefficients. Note that this improved collision matrix is still invertible. Through the Chapman-Enskog (CE) analysis and to recover the correct Newtonian viscous stress (see Supplemental Material for details [25]), the coefficients in $\mathbf{m}^{\mathrm{eq}}$ and $\mathbf{S}$ should satisfy

$$
\begin{gathered}
\alpha_{2}=-\frac{2 \alpha_{1}+\varpi+1}{1-\varpi}, \quad \beta_{2}=-\frac{2 \beta_{1}}{1-\varpi}, \\
k=1-\varpi, \quad h=\frac{6 \varpi(1-\varpi)}{1-3 \varpi}, \quad b=\frac{1-\varpi}{1-3 \varpi},
\end{gathered}
$$

where $\varpi$ is related to the bulk viscosity. The recovered macroscopic equation at the Navier-Stokes level is

$$
\left\{\begin{array}{l}
\partial_{t} \rho+\nabla \cdot(\rho \mathbf{u})=0, \\
\partial_{t}(\rho \mathbf{u})+\nabla \cdot(\rho \mathbf{u u})=-\nabla p_{\mathrm{LBE}}+\mathbf{F}+\nabla \cdot \mathbf{\Pi}+O\left(M a^{3}\right),
\end{array}\right.
$$

where $p_{\mathrm{LBE}}$ is the recovered EOS, $\boldsymbol{\Pi}=\rho \nu[\nabla \mathbf{u}+\mathbf{u} \nabla-$ $(\nabla \cdot \mathbf{u}) \mathbf{I}]+\rho \varsigma(\nabla \cdot \mathbf{u}) \mathbf{I}$ is the recovered Newtonian viscous stress, and $M a$ denotes the Mach number. Here, the EOS $p_{\mathrm{LBE}}$, the kinematic viscosity $\nu$ and the bulk viscosity $\varsigma$ are expressed as

$$
\begin{gathered}
p_{\mathrm{LBE}}=c_{s}^{2}\left[\left(2+\alpha_{1}\right) \rho+\beta_{1} \eta\right], \\
\nu=c_{s}^{2}\left(s_{p}^{-1}-\frac{1}{2}\right) \delta_{t}, \quad \varsigma=\varpi c_{s}^{2}\left(s_{e}^{-1}-\frac{1}{2}\right) \delta_{t},
\end{gathered}
$$

with lattice sound speed $c_{s}=c / \sqrt{3}$. Obviously, $p_{\mathrm{LBE}}$ can be arbitrarily tuned via the built-in $\eta$. Before proceeding further, some discussion on the present LB model with self-tuning EOS is useful. For the coefficients $\alpha_{1,2}$ and $\beta_{1,2}, \alpha_{1}=-1$ and $\beta_{1}=1$ are set as ordinary, and one has $\alpha_{2}=1$. Therefore, $\mathbf{m}^{\text {eq }}$ given by Eq. (3) can be decomposed into the ordinary one derived from the Hermite expansion of the Maxwell-Boltzmann distribution and the extra one related to $\eta$. The coefficient $\varpi$ plays an important role here. When $\varpi=1$, one has $\beta_{2} \rightarrow \infty, k=0, h=0$, and $b=0$. Thus, $\eta$ should be set to 0 to avoid singularity, implying that the present model degenerates into the classical LB model with idealgas EOS. When $\varpi=1 / 3$, one has $\beta_{2}=-3, k=2 / 3$, $h \rightarrow \infty$, and $b \rightarrow \infty$. Thus, the velocity-dependent terms in $\mathbf{S}$ should be removed to avoid singularity, which means that Newtonian viscous stress cannot be recovered and Galilean invariance is lost. Compared with previous LB models derived from the Enskog equation via systematic discretization procedures [6-8], the present model is directly constructed at the discrete level in moment space and thus is free of complicated derivative terms, which trigger numerical instability and restrict real applications of previous models $[18,19]$.

In the macroscopic equation recovered at the NavierStokes level [Eq. (8)], some cubic terms of velocity exist, which are usually ignored under the low Mach number condition in LB method. By retaining the high-order terms of velocity in $\mathbf{m}^{\mathrm{eq}}$ and $\mathbf{F}_{m}$ [Eqs. (3) and (4)], the additional cubic terms can be partially eliminated [26]. To eliminate the remaining cubic terms, the collision process described by Eq. (2) can be improved as

$$
\begin{array}{r}
\overline{\mathbf{m}}=\mathbf{m}+\delta_{t} \mathbf{F}_{m}-\mathbf{S}\left(\mathbf{m}-\mathbf{m}^{\mathrm{eq}}+\frac{\delta_{t}}{2} \mathbf{F}_{m}\right) \\
-\mathbf{R}\left(\mathbf{I}-\frac{\mathbf{S}}{2}\right)\left(\mathbf{m}-\mathbf{m}^{\mathrm{eq}}+\frac{\delta_{t}}{2} \mathbf{F}_{m}\right) \\
-\delta_{x} \mathbf{T} \cdot \nabla \rho-\frac{\delta_{x}}{c^{2}} \mathbf{X} \cdot \nabla p_{\mathrm{LBE}},
\end{array}
$$

where $\mathbf{R}, \mathbf{T}$ and $\mathbf{X}$ are $9 \times 9,9 \times 2$ and $9 \times 2$ matrices of order $M a^{2}, M a^{3}$ and $M a^{3}$, respectively, and thus the corresponding terms have negligible effects on the numerical stability. These correction matrices are set in the following forms [27]

$$
\mathbf{R}=\left[\begin{array}{ccccccccc}
0 & 0 & 0 & 0 & 0 & 0 & 0 & 0 & 0 \\
0 & R_{11} & 0 & 0 & 0 & 0 & 0 & R_{17} & R_{18} \\
0 & 0 & 0 & 0 & 0 & 0 & 0 & 0 & 0 \\
0 & 0 & 0 & 0 & 0 & 0 & 0 & 0 & 0 \\
0 & 0 & 0 & 0 & 0 & 0 & 0 & 0 & 0 \\
0 & 0 & 0 & 0 & 0 & 0 & 0 & 0 & 0 \\
0 & 0 & 0 & 0 & 0 & 0 & 0 & 0 & 0 \\
0 & R_{71} & 0 & 0 & 0 & 0 & 0 & R_{77} & R_{78} \\
0 & R_{81} & 0 & 0 & 0 & 0 & 0 & R_{87} & R_{88}
\end{array}\right]
$$




$$
\begin{aligned}
& \mathbf{T}=\left(\mathbf{0}, \mathbf{T}_{1}, \mathbf{0}, \mathbf{0}, \mathbf{0}, \mathbf{0}, \mathbf{0}, \mathbf{T}_{7}, \mathbf{T}_{8}\right)^{\mathrm{T}} \\
& \mathbf{X}=\left(\mathbf{0}, \mathbf{X}_{1}, \mathbf{0}, \mathbf{0}, \mathbf{0}, \mathbf{0}, \mathbf{0}, \mathbf{X}_{7}, \mathbf{X}_{8}\right)^{\mathrm{T}}
\end{aligned}
$$

Through the CE analysis, the nonzero elements in Eq. (11) can be uniquely and locally determined, and the truncated term in Eq. (8) is improved from $O\left(M a^{3}\right)$ to $O\left(M a^{5}\right)$ (see Supplemental Material for details [25]).

As analyzed by He and Doolen [8], a thermodynamically consistent kinetic model for multiphase flows can be established by combining Enskog theory for dense gases and mean-field theory for long-range molecular interaction. In the Enskog equation, short-range molecular interaction is considered by the collision term, and consequently nonideal-gas EOS is recovered [24]. From this viewpoint, the present LB model with self-tuning EOS can be interpreted as the incorporation of short-range molecular interaction, and thus the long-range molecular interaction remains to be included to construct a valid model for multiphase flows. Following the idea of the pseudopotential LB model $[4,9]$, a pairwise interaction force is introduced to mimic the long-range molecular interaction. Here, nearest-neighbor interaction is considered, and the interaction force is given as

$$
\mathbf{F}(\mathbf{x})=G^{2} \rho(\mathbf{x}) \sum_{i} \omega\left(\left|\mathbf{e}_{i} \delta_{t}\right|^{2}\right) \rho\left(\mathbf{x}+\mathbf{e}_{i} \delta_{t}\right) \mathbf{e}_{i} \delta_{t},
$$

where $G^{2}$ is used to control the interaction strength, and the weights $\omega\left(\delta_{x}^{2}\right)=1 / 3$ and $\omega\left(2 \delta_{x}^{2}\right)=1 / 12$ to maximize the isotropy degree of $\mathbf{F}$. Note that Eq. (12) implies that the long-range molecular interaction is attractive.

The interaction force given by Eq. (12) is incorporated into the LB equation via the discrete force term. Based on our previous analysis [28], some $\varepsilon^{3}$-order terms will be caused by the discrete lattice effect on the force term, which should be considered for multiphase flows. In order to cancel such effects, a consistent scheme for the $\varepsilon^{3}$-order additional term is employed. The collision process described by Eq. (10) is further improved as

$$
\begin{array}{r}
\overline{\mathbf{m}}=\mathbf{m}+\delta_{t} \mathbf{F}_{m}-\mathbf{S}\left(\mathbf{m}-\mathbf{m}^{\mathrm{eq}}+\frac{\delta_{t}}{2} \mathbf{F}_{m}\right)+\mathbf{S Q}_{m} \\
-\mathbf{R}\left(\mathbf{I}-\frac{\mathbf{S}}{2}\right)\left(\mathbf{m}-\mathbf{m}^{\mathrm{eq}}+\frac{\delta_{t}}{2} \mathbf{F}_{m}\right) \\
-\delta_{x} \mathbf{T} \cdot \nabla \rho-\frac{\delta_{x}}{c^{2}} \mathbf{X} \cdot \nabla p_{\mathrm{LBE}},
\end{array}
$$

where the discrete additional term $\mathbf{Q}_{m}$ is set as

$$
\mathbf{Q}_{m}=\left(0, \frac{|\tilde{\mathbf{F}}|^{2}}{2},-\frac{|\tilde{\mathbf{F}}|^{2}}{2}, 0,0,0,0, \frac{\tilde{F}_{x}^{2}-\tilde{F}_{y}^{2}}{12}, \frac{\tilde{F}_{x} \tilde{F}_{y}}{12}\right)^{\mathrm{T}},
$$

and $\tilde{\mathbf{F}}=\mathbf{F} /(G \rho c)$. In the CE analysis, $\mathbf{F}$ is of order $\varepsilon^{1}$, and thus $\mathbf{Q}_{m}$ is of order $\varepsilon^{2}$. Through the third-order CE analysis, the following macroscopic equation in steady and stationary situation can be recovered (see Supplemental Material for details [25])

$$
\left\{\begin{array}{l}
\partial_{t} \rho=0, \\
\partial_{t}(\rho \mathbf{u})=-\nabla p_{\mathrm{LBE}}+\mathbf{F}+\mathbf{R}_{\text {iso }}+\mathbf{R}_{\text {add }}+\overline{\mathbf{R}}_{\text {iso }}+\overline{\mathbf{R}}_{\text {aniso }},
\end{array}\right.
$$

where $\mathbf{R}_{\text {iso }}=\frac{1}{12} \delta_{x}^{2} \nabla \cdot \nabla \mathbf{F}$ is the isotropic term caused by the discrete lattice effect, $\mathbf{R}_{\text {add }}=-\frac{1}{24} c^{2} \nabla \cdot[2 \tilde{\mathbf{F}} \tilde{\mathbf{F}}+(\tilde{\mathbf{F}} \cdot \tilde{\mathbf{F}}) \mathbf{I}]$ is the additional term introduced by $\mathbf{S Q}_{m}$ to cancel the effect of $\mathbf{R}_{\text {iso }}, \overline{\mathbf{R}}_{\text {iso }}=-\frac{1}{6} \delta_{x}^{2}\left[(k+1) \tau_{e} \tau_{q}-\tau_{p} \tau_{q}\right] \nabla \cdot \nabla \nabla \bar{p}$ and $\overline{\mathbf{R}}_{\text {aniso }}=-\frac{1}{12} \delta_{x}^{2}\left(12 \tau_{p} \tau_{q}-1\right)\left(\partial_{y}^{2} \partial_{x} \bar{p}, \partial_{x}^{2} \partial_{y} \bar{p}\right)^{\mathrm{T}}$ are the isotropic and anisotropic terms caused by achieving selftuning EOS, respectively. Here, $\tau_{e, p, q}=s_{e, p, q}^{-1}-1 / 2$ and $\bar{p}=\left(3+\beta_{2}\right) c_{s}^{2} \eta$. Note that $\mathbf{R}_{\text {iso }}, \mathbf{R}_{\text {add }}, \overline{\mathbf{R}}_{\text {iso }}$ and $\overline{\mathbf{R}}_{\text {aniso }}$ are all recovered at the $\varepsilon^{3}$-order and thus disappear from the macroscopic equation at the Navier-Stokes level. For multiphase flows, $\overline{\mathbf{R}}_{\text {iso }}$ and $\overline{\mathbf{R}}_{\text {aniso }}$ should be eliminated by setting

$$
\tau_{p} \tau_{q}=(k+1) \tau_{e} \tau_{q}=1 / 12,
$$

and $\mathbf{R}_{\text {iso }}$ and $\mathbf{R}_{\text {add }}$ can be absorbed into the pressure tensor. Therefore, the pressure tensor recovered by the present model is defined as $\nabla \cdot \mathbf{P}=\nabla p_{\mathrm{LBE}}-\mathbf{F}-\mathbf{R}_{\text {iso }}-$ $\mathbf{R}_{\text {add }}$. By performing Taylor series expansion of the interaction force, the following pressure tensor can be derived (see Supplemental Material for details [25])

$$
\mathbf{P}=\left(p_{\mathrm{EOS}}-\kappa \rho \nabla \cdot \nabla \rho-\frac{\kappa}{2} \nabla \rho \cdot \nabla \rho\right) \mathbf{I}+\kappa \nabla \rho \nabla \rho,
$$

where $\kappa=G^{2} \delta_{x}^{4} / 4$ and the EOS is

$$
p_{\mathrm{EOS}}=p_{\mathrm{LBE}}-\frac{G^{2} \delta_{x}^{2}}{2} \rho^{2} .
$$

Obviously, $\mathbf{P}$ given by Eq. (17) is naturally consistent with thermodynamic theory [29], where the free energy $\Psi$ is defined as

$$
\Psi=\int_{V}\left(\psi_{b}+\frac{\kappa}{2}|\nabla \rho|^{2}\right) d \mathbf{x} .
$$

Here, $\psi_{b}$ is the bulk free-energy density related to EOS $p_{\mathrm{EOS}}=\rho \partial_{\rho} \psi_{b}-\psi_{b}$, and $\frac{\kappa}{2}|\nabla \rho|^{2}$ is the interfacial freeenergy density. Based on Eq. (17), the Maxwell construction can be derived.

In this work, the Carnahan-Starling EOS [30] is taken as an example

$$
p_{\mathrm{EOS}}=K_{\mathrm{EOS}}\left[\rho R T \frac{1+\vartheta+\vartheta^{2}-\vartheta^{3}}{(1-\vartheta)^{3}}-a \rho^{2}\right],
$$

where $R$ is the gas constant, $T$ is the temperature, $\vartheta=b \rho / 4, a=0.496388 R^{2} T_{c}^{2} / p_{c}$ and $b=0.187295 R T_{c} / p_{c}$ with $T_{c}$ and $p_{c}$ denoting the critical temperature and pressure, respectively. The scaling factor $K_{\mathrm{EOS}}$ [31] is introduced here to adjust the magnitude of bulk free-energy density $\psi_{b}$. In the Carnahan-Starling EOS, the first and second terms describe the effects of short-range (repulsive) and long-range (attractive) molecular interactions, respectively [30]. Therefore, a consistency between Eqs. (18) and (20) can be established, and then the interaction strength is set as

$$
G=K_{\mathrm{INT}} \sqrt{2 K_{\mathrm{EOS}} a / \delta_{x}^{2}},
$$


where the scaling factor $K_{\mathrm{INT}}$ is introduced to adjust the interfacial free-energy density $\frac{\kappa}{2}|\nabla \rho|^{2}$, and the lattice sound speed is chosen as

$$
c_{s}=\left.K_{\mathrm{INT}} \sqrt{\partial_{\rho}\left(p_{\mathrm{EOS}}+K_{\mathrm{EOS}} a \rho^{2}\right)}\right|_{\rho=\rho_{l}} .
$$

With this configuration, it is known from thermodynamic theory that the surface tension $\sigma$ and interface thickness $W$ satisfy

$$
\sigma \propto K_{\mathrm{EOS}} K_{\mathrm{INT}}, \quad W \propto K_{\mathrm{INT}},
$$

which have also been numerically validated (see Supplemental Material for details [25]), and where the proportionality constants can be analytically determined by the pressure tensor. Thus, in real applications of the present LB model, the surface tension and interface thickness can be independently prescribed.

Simulations are performed with $\varpi=1 / 6, a=1, b=4$, $R=1$, and $\delta_{x}=1$, and a detailed implementation of the collision process [Eq. (13)] is given in Supplemental Material [25]. The coexistence curve, as a function of reduced temperature $T_{r}$, is firstly computed by simulating a flat interface on a $1024 \delta_{x} \times 4 \delta_{x}$ domain, as shown in Fig. 1 . It can be seen that the numerical result agrees well with the thermodynamic result by Maxwell construction. When $T_{r}<0.6$, there exists slight deviation in the gas branch, which is caused by the spatial discretization error in the interfacial region and can be reduced by increasing the interface thickness. A liquid droplet is then simulated with various $K_{\mathrm{EOS}}$ and $K_{\mathrm{INT}}$ on a $1024 \delta_{x} \times 1024 \delta_{x}$ domain with the droplet diameter being $512 \delta_{x}$. Accordingly, the surface tension is calculated via Laplace's law and the interface thickness is measured from $\rho=0.95 \rho_{g}+0.05 \rho_{l}$ to $0.05 \rho_{g}+0.95 \rho_{l}$. Proportionalities described by Eq. (23) can be accurately observed, and the proportionality constants are in good agreement with $\sigma$ and $W$ predicted by $\mathbf{P}$ with $K_{\mathrm{EOS}}=K_{\mathrm{INT}}=1$ for flat interface, as shown in Fig. 2.

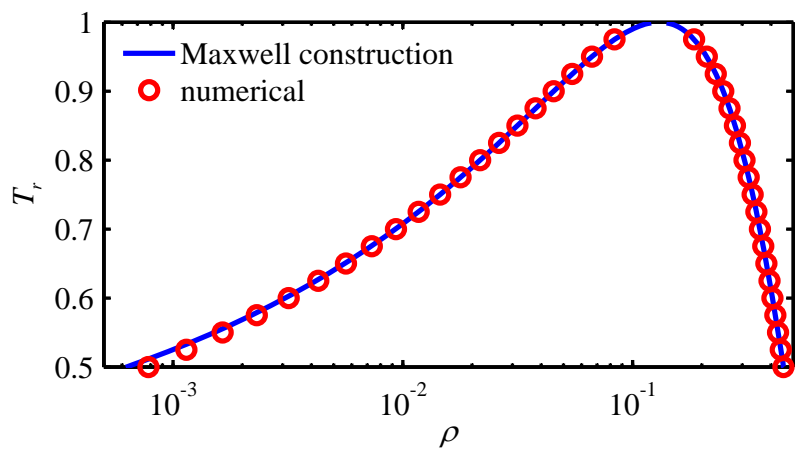

FIG. 1. Coexistence curves obtained by simulation of flat interface and predicted by Maxwell construction.

As a dynamic problem, oscillation of an elliptic droplet with the semi-major and minor axes being $96.0 \delta_{x}$ and

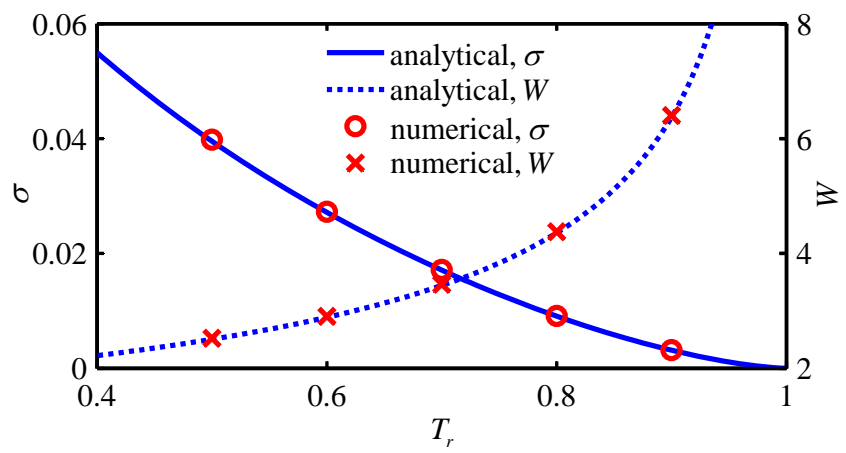

FIG. 2. Proportionality constants in Eq. (23) obtained by simulation of liquid droplet with various $K_{\mathrm{EOS}}$ and $K_{\mathrm{INT}}$ and predicted by pressure tensor with $K_{\mathrm{EOS}}=K_{\mathrm{INT}}=1$ for flat interface.

$42.7 \delta_{x}$ is simulated on a $512 \delta_{x} \times 512 \delta_{x}$ domain. Here, $T_{r}=0.6, \sigma=0.01$, and $W=10$ are chosen. The oscillation period, numerically measured when the oscillation becomes weak enough, is $18346 \delta_{t}$, which agrees well with the analytical solution $18628.0 \delta_{t}$ [35]. Headon collision of equal-sized droplets is further simulated with $T_{r}=0.7, \sigma=0.01$, and $W=10$. The computational domain size is $1024 \times 1024$, and the droplet diameter is $128 \delta_{x}$. The head-on collision outcome is mainly controlled by the Weber number $W e=\rho_{l} U^{2} D / \sigma$ and Reynolds number $R e=U D / \nu$, with $U$ and $D$ denoting the relative velocity and droplet diameter, respectively. All four regimes for head-on collision, experimentally observed by Qian and Law [32], are successfully reproduced here, as shown in Fig. 3. For $W e=0.01$ and $R e=1$, the droplets approach each other and then merge with small deformation. As $W e$ increases to 0.1 , the droplets bounce back without merging. Here, it is interesting to note that this "bouncing" regime has not been observed in previous simulations by the pseudopotential and free-energy LB models [33, 34]. For $W e=20$ and $R e=100$, merging happens again, accompanied with large deformation in this regime. For $W e=60$ and $R e=200$, the outward motion caused by strong impact splits the merged mass into three parts, with two main droplets separating from both sides and a satellite droplet residing at the center, as shown in Fig. 3(d).

In summary, we have developed a novel LB model for multiphase flows, which complies with the thermodynamic foundations of kinetic theory and thus is naturally consistent with thermodynamic theory. The underlying short-range and long-range molecular interactions are separately incorporated by constructing an LB model with self-tuning EOS and introducing a pairwise interaction force. The present model combines the advantages of the popular pseudopotential and free-energy LB models. Most computations can be carried out locally, and the surface tension and interface thickness can be independently prescribed in real applications. 

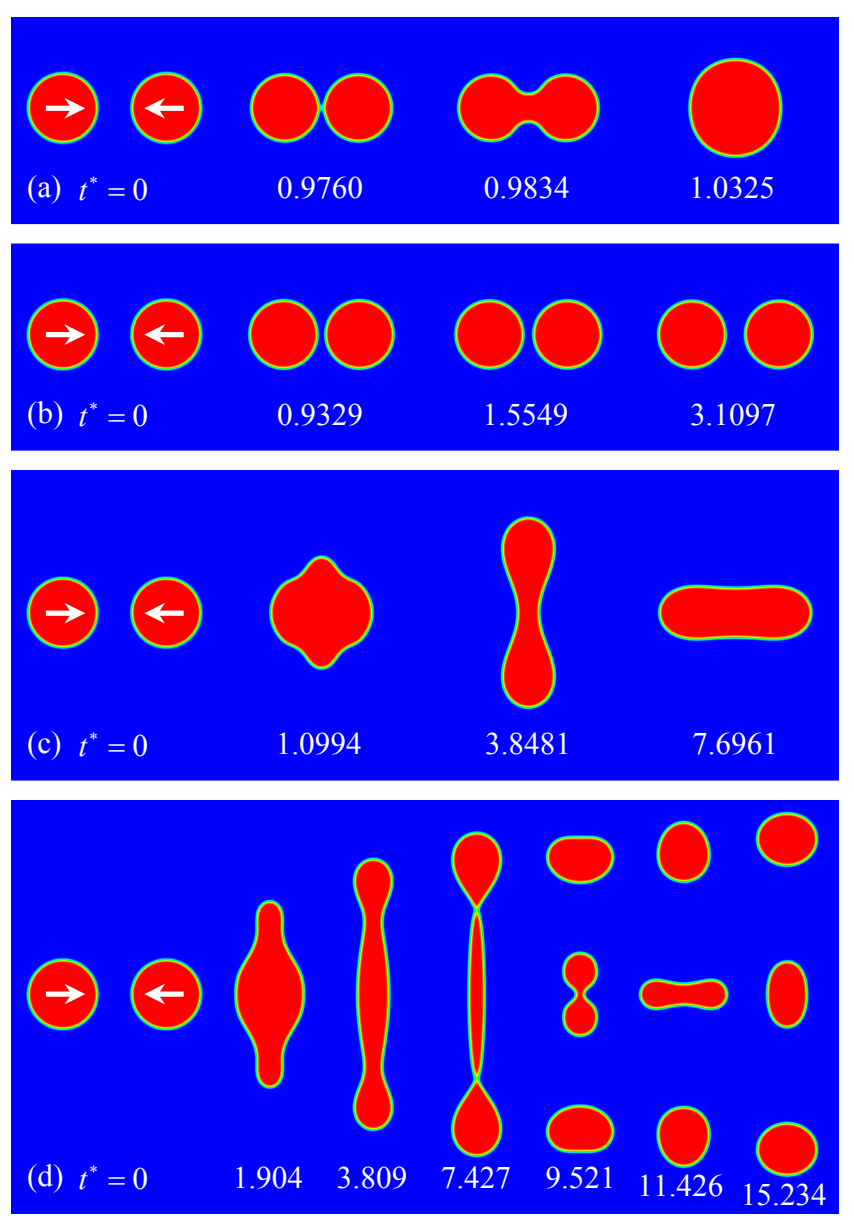

FIG. 3. Head-on collision processes of equal-sized droplets at different dimensionless time $t^{*}=t U / D$ with (a) $W e=0.01$ and $R e=1$, (b) $W e=0.1$ and $R e=1$, (c) $W e=20$ and $R e=100$, and (d) $W e=60$ and $R e=200$.

R.H. acknowledges the support by the Alexander von Humboldt Foundation, Germany. This work was also supported by the National Natural Science Foundation of China through Grant No. 51536005.

* rongzong.huang@tum.de

† whysrj@sjtu.edu.cn

‡ nikolaus.adams@tum.de

[1] G. R. McNamara and G. Zanetti, Phys. Rev. Lett. 61, 2332 (1988).

[2] A. K. Gunstensen, D. H. Rothman, S. Zaleski, and G. Zanetti, Phys. Rev. A 43, 4320 (1991).

[3] Q. Li, K. H. Luo, Q. J. Kang, Y. L. He, Q. Chen, and Q. Liu, Prog. Energy Combust. Sci. 52, 62 (2016).

[4] X. Shan and H. Chen, Phys. Rev. E 47, 1815 (1993).

[5] M. R. Swift, W. R. Osborn, and J. M. Yeomans, Phys. Rev. Lett. 75, 830 (1995).

[6] X. He, X. Shan, and G. D. Doolen, Phys. Rev. E 57, R13 (1998).
[7] L.-S. Luo, Phys. Rev. Lett. 81, 1618 (1998).

[8] X. He and G. D. Doolen, J. Stat. Phys. 107, 309 (2002).

[9] X. Shan and H. Chen, Phys. Rev. E 49, 2941 (1994).

[10] M. Sbragaglia, R. Benzi, L. Biferale, S. Succi, K. Sugiyama, and F. Toschi, Phys. Rev. E 75, 026702 (2007).

[11] Q. Li, K. H. Luo, and X. J. Li, Phys. Rev. E 86, 016709 (2012).

[12] S. Khajepor, J. Wen, and B. Chen, Phys. Rev. E 91, 023301 (2015).

[13] M. R. Swift, E. Orlandini, W. R. Osborn, and J. M. Yeomans, Phys. Rev. E 54, 5041 (1996).

[14] D. J. Holdych, D. Rovas, J. G. Georgiadis, and R. O. Buckius, Int. J. Mod. Phys. C 9, 1393 (1998).

[15] T. Inamuro, N. Konishi, and F. Ogino, Comput. Phys. Commun. 129, 32 (2000).

[16] A. N. Kalarakis, V. N. Burganos, and A. C. Payatakes, Phys. Rev. E 65, 056702 (2002).

[17] A. N. Kalarakis, V. N. Burganos, and A. C. Payatakes, Phys. Rev. E 67, 016702 (2003).

[18] X. He, S. Chen, and R. Zhang, J. Comput. Phys. 152, 642 (1999).

[19] T. Lee and C.-L. Lin, J. Comput. Phys. 206, 16 (2005).

[20] M. E. McCracken and J. Abraham, Phys. Rev. E 71, 036701 (2005).

[21] P. Lallemand and L.-S. Luo, Phys. Rev. E 61, 6546 (2000).

[22] Y. H. Qian, D. d'Humières, and P. Lallemand, Europhys. Lett. 17, 479 (1992).

[23] P. J. Dellar, Phys. Rev. E 65, 036309 (2002).

[24] S. Chapman and T. G. Cowling, The Mathematical Theory of Non-Uniform Gases, 3rd ed. (Cambridge University Press, Cambridge, 1970).

[25] See Supplemental Material for details.

[26] P. J. Dellar, J. Comput. Phys. 259, 270 (2014).

[27] R. Huang, H. Wu, and N. A. Adams, Phys. Rev. E 97, 053308 (2018).

[28] R. Huang and H. Wu, J. Comput. Phys. 327, 121 (2016).

[29] J. S. Rowlinson and B. Widom, Molecular Theory of Capillarity (Oxford University Press, Oxford, 1982).

[30] N. F. Carnahan and K. E. Starling, J. Chem. Phys. 51, 635 (1969).

[31] A. J. Wagner and C. M. Pooley, Phys. Rev. E 76, 045702 (2007).

[32] J. Qian and C. K. Law, J. Fluid Mech. 331, 59 (1997).

[33] D. Lycett-Brown, K. H. Luo, R. Liu, and P. Lv, Phys. Fluids 26, 023303 (2014).

[34] A. M. Moqaddam, S. S. Chikatamarla, and I. V. Karlin, Phys. Fluids 28, 022106 (2016).

[35] The analytical solution of the oscillation period is calculated via $2 \pi\left[6 \sigma /\left(\rho_{l} R_{0}^{3}\right)\right]^{-1 / 2}[36]$, where the surface tension $\sigma$, the liquid density $\rho_{l}$, and the equilibrium radius $R_{0}$ are numerically measured when the oscillation finally stops.

[36] Q. Li, K. H. Luo, and X. J. Li, Phys. Rev. E 87, 053301 (2013). 


\title{
Supplemental Material \\ Lattice Boltzmann model with self-tuning equation of state for multiphase flows
}

\author{
Rongzong Huang, ${ }^{1,2, *}$ Huiying $\mathrm{Wu},{ }^{1, \dagger}$ and Nikolaus A. Adams ${ }^{2}, \ddagger$ \\ ${ }^{1}$ School of Mechanical Engineering, Shanghai Jiao Tong University, 200240 Shanghai, China \\ ${ }^{2}$ Institute of Aerodynamics and Fluid Mechanics, \\ Technical University of Munich, 85748 Garching, Germany
}

(Dated: September 7, 2018)

In this Supplemental Material, theoretical analysis of the present LB model for multiphase flows is provided in detail. We start from the collision process given by Eq. (13) in the main text. Both the cubic terms of velocity at the Navier-Stokes level $\left(\varepsilon^{2}\right.$-order $)$ and the additional term at the $\varepsilon^{3}$-order are considered. The collision process, calculated at position $\mathbf{x}$ and time $t$ in moment space, is given as

$$
\overline{\mathbf{m}}=\mathbf{m}+\delta_{t} \mathbf{F}_{m}-\mathbf{S}\left(\mathbf{m}-\mathbf{m}^{\mathrm{eq}}+\frac{\delta_{t}}{2} \mathbf{F}_{m}\right)+\mathbf{S Q}_{m}-\mathbf{R}\left(\mathbf{I}-\frac{\mathbf{S}}{2}\right)\left(\mathbf{m}-\mathbf{m}^{\mathrm{eq}}+\frac{\delta_{t}}{2} \mathbf{F}_{m}\right)-\delta_{x} \mathbf{T} \cdot \nabla \rho-\frac{\delta_{x}}{c^{2}} \mathbf{X} \cdot \nabla p_{\mathrm{LBE}},
$$

and the streaming process, executed in discrete velocity space, is

$$
f_{i}\left(\mathbf{x}+\mathbf{e}_{i} \delta_{t}, t+\delta_{t}\right)=\bar{f}_{i}(\mathbf{x}, t) .
$$

The discrete velocity for D2Q9 lattice is [1]

$$
\mathbf{e}_{i}= \begin{cases}c(0,0)^{\mathrm{T}}, & i=0, \\ c(\cos [(i-1) \pi / 2], \sin [(i-1) \pi / 2])^{\mathrm{T}}, & i=1,2,3,4, \\ \sqrt{2} c(\cos [(2 i-1) \pi / 4], \sin [(2 i-1) \pi / 4])^{\mathrm{T}}, & i=5,6,7,8,\end{cases}
$$

and the corresponding dimensionless transformation matrix is $[2]$

$$
\mathbf{M}=\left[\begin{array}{ccccccccc}
1 & 1 & 1 & 1 & 1 & 1 & 1 & 1 & 1 \\
-4 & -1 & -1 & -1 & -1 & 2 & 2 & 2 & 2 \\
4 & -2 & -2 & -2 & -2 & 1 & 1 & 1 & 1 \\
0 & 1 & 0 & -1 & 0 & 1 & -1 & -1 & 1 \\
0 & -2 & 0 & 2 & 0 & 1 & -1 & -1 & 1 \\
0 & 0 & 1 & 0 & -1 & 1 & 1 & -1 & -1 \\
0 & 0 & -2 & 0 & 2 & 1 & 1 & -1 & -1 \\
0 & 1 & -1 & 1 & -1 & 0 & 0 & 0 & 0 \\
0 & 0 & 0 & 0 & 0 & 1 & -1 & 1 & -1
\end{array}\right] .
$$

\section{CHAPMAN-ENSKOG EXPANSION}

Performing Taylor series expansion of $f_{i}\left(\mathbf{x}+\mathbf{e}_{i} \delta_{t}, t+\delta_{t}\right)$ centered at $(\mathbf{x}, t)$ in Eq. (2), and then transforming the result into moment space and combining it with Eq. (1), we can obtain

$$
\begin{aligned}
\left(\mathbf{I} \partial_{t}+\mathbf{D}\right) \mathbf{m} & +\frac{\delta_{t}}{2}\left(\mathbf{I} \partial_{t}+\mathbf{D}\right)^{2} \mathbf{m}+\frac{\delta_{t}^{2}}{6}\left(\mathbf{I} \partial_{t}+\mathbf{D}\right)^{3} \mathbf{m}-\mathbf{F}_{m}+O\left(\delta_{t}^{3}\right) \\
& =-\frac{\mathbf{S}}{\delta_{t}}\left(\mathbf{m}-\mathbf{m}^{\mathrm{eq}}+\frac{\delta_{t}}{2} \mathbf{F}_{m}\right)+\frac{\mathbf{S}}{\delta_{t}} \mathbf{Q}_{m}-\frac{\mathbf{R}}{\delta_{t}}\left(\mathbf{I}-\frac{\mathbf{S}}{2}\right)\left(\mathbf{m}-\mathbf{m}^{\mathrm{eq}}+\frac{\delta_{t}}{2} \mathbf{F}_{m}\right)-\frac{\delta_{x} \mathbf{T}}{\delta_{t}} \cdot \nabla \rho-\frac{\delta_{x} \mathbf{X}}{c^{2} \delta_{t}} \cdot \nabla p_{\mathrm{LBE}},
\end{aligned}
$$

where $\mathbf{D}=\mathbf{M}\left[\operatorname{diag}\left(\mathbf{e}_{i} \cdot \nabla\right)\right] \mathbf{M}^{-1}$. In this work, the following classical Chapman-Enskog expansions are adopted [3]

$$
\partial_{t}=\sum_{n=1}^{+\infty} \varepsilon^{n} \partial_{t n}, \quad \nabla=\varepsilon^{1} \nabla_{1}, \quad f_{i}=\sum_{n=0}^{+\infty} \varepsilon^{n} f_{i}^{(n)}, \quad \mathbf{F}=\varepsilon^{1} \mathbf{F}^{(1)},
$$

\footnotetext{
* rongzong.huang@tum.de

$\dagger$ whysrj@sjtu.edu.cn

‡nikolaus.adams@tum.de
} 
which indicate

$$
\mathbf{D}=\varepsilon^{1} \mathbf{D}_{1}, \quad \mathbf{m}=\sum_{n=0}^{+\infty} \varepsilon^{n} \mathbf{m}^{(n)}, \quad \mathbf{F}_{m}=\varepsilon^{1} \mathbf{F}_{m}^{(1)}, \quad \mathbf{Q}_{m}=\varepsilon^{2} \mathbf{Q}_{m}^{(2)} .
$$

Here, $\varepsilon$ is the small expansion parameter. Substituting the above expansions into Eq. (5), we can rewrite Eq. (5) in the consecutive orders of $\varepsilon$ as follows

$$
\begin{aligned}
& \varepsilon^{0}:-\frac{\mathbf{S}}{\delta_{t}}\left(\mathbf{m}^{(0)}-\mathbf{m}^{\mathrm{eq}}\right)-\frac{\mathbf{R}}{\delta_{t}}\left(\mathbf{I}-\frac{\mathbf{S}}{2}\right)\left(\mathbf{m}^{(0)}-\mathbf{m}^{\mathrm{eq}}\right)=\mathbf{0}, \\
& \varepsilon^{1}:\left(\mathbf{I} \partial_{t 1}+\mathbf{D}_{1}\right) \mathbf{m}^{(0)}-\mathbf{F}_{m}^{(1)}=-\frac{\mathbf{S}}{\delta_{t}}\left(\mathbf{m}^{(1)}+\frac{\delta_{t}}{2} \mathbf{F}_{m}^{(1)}\right)-\frac{\mathbf{R}}{\delta_{t}}\left(\mathbf{I}-\frac{\mathbf{S}}{2}\right)\left(\mathbf{m}^{(1)}+\frac{\delta_{t}}{2} \mathbf{F}_{m}^{(1)}\right)-\frac{\delta_{x} \mathbf{T}}{\delta_{t}} \cdot \nabla_{1} \rho-\frac{\delta_{x} \mathbf{X}}{c^{2} \delta_{t}} \cdot \nabla_{1} p_{\mathrm{LBE}} \\
& \varepsilon^{2}: \partial_{t 2} \mathbf{m}^{(0)}+\left(\mathbf{I} \partial_{t 1}+\mathbf{D}_{1}\right) \mathbf{m}^{(1)}+\frac{\delta_{t}}{2}\left(\mathbf{I} \partial_{t 1}+\mathbf{D}_{1}\right)^{2} \mathbf{m}^{(0)}=-\frac{\mathbf{S}}{\delta_{t}} \mathbf{m}^{(2)}+\frac{\mathbf{S}}{\delta_{t}} \mathbf{Q}_{m}^{(2)}-\frac{\mathbf{R}}{\delta_{t}}\left(\mathbf{I}-\frac{\mathbf{S}}{2}\right) \mathbf{m}^{(2)}, \\
& \varepsilon^{3}: \partial_{t 3} \mathbf{m}^{(0)}+\partial_{t 2} \mathbf{m}^{(1)}+\left(\mathbf{I} \partial_{t 1}+\mathbf{D}_{1}\right) \mathbf{m}^{(2)}+\delta_{t}\left(\mathbf{I} \partial_{t 1}+\mathbf{D}_{1}\right) \partial_{t 2} \mathbf{m}^{(0)} \\
& +\frac{\delta_{t}}{2}\left(\mathbf{I} \partial_{t 1}+\mathbf{D}_{1}\right)^{2} \mathbf{m}^{(1)}+\frac{\delta_{t}^{2}}{6}\left(\mathbf{I} \partial_{t 1}+\mathbf{D}_{1}\right)^{3} \mathbf{m}^{(0)}=-\frac{\mathbf{S}}{\delta_{t}} \mathbf{m}^{(3)}-\frac{\mathbf{R}}{\delta_{t}}\left(\mathbf{I}-\frac{\mathbf{S}}{2}\right) \mathbf{m}^{(3)} .
\end{aligned}
$$

\section{SECOND-ORDER ANALYSIS}

Firstly, the standard second-order analysis is carried out to recover the macroscopic equation at the Navier-Stokes level ( $\varepsilon^{2}$-order). To simplify the descriptions, we introduce the following notations

$$
\mathbf{G}^{(1)}=\mathbf{m}^{(1)}+\frac{\delta_{t}}{2} \mathbf{F}_{m}^{(1)}, \quad \hat{\mathbf{G}}^{(1)}=\left(\mathbf{I}-\frac{\mathbf{S}}{2}\right) \mathbf{G}^{(1)}, \quad \tilde{\mathbf{G}}^{(1)}=\left(\mathbf{I}-\frac{\mathbf{R}}{2}\right) \hat{\mathbf{G}}^{(1)}-\frac{\delta_{x} \mathbf{T}}{2} \cdot \nabla_{1} \rho-\frac{\delta_{x} \mathbf{X}}{2 c^{2}} \cdot \nabla_{1} p_{\mathrm{LBE}} .
$$

Therefore, the $\varepsilon^{0}-, \varepsilon^{1}$-, and $\varepsilon^{2}$-order equations in Eq. (7) can be reformulated as

$$
\begin{gathered}
\varepsilon^{0}:-\frac{\mathbf{S}}{\delta_{t}}\left(\mathbf{m}^{(0)}-\mathbf{m}^{\mathrm{eq}}\right)-\frac{\mathbf{R}}{\delta_{t}}\left(\mathbf{I}-\frac{\mathbf{S}}{2}\right)\left(\mathbf{m}^{(0)}-\mathbf{m}^{\mathrm{eq}}\right)=\mathbf{0}, \\
\varepsilon^{1}:\left(\mathbf{I} \partial_{t 1}+\mathbf{D}_{1}\right) \mathbf{m}^{(0)}-\mathbf{F}_{m}^{(1)}=-\frac{\mathbf{S}}{\delta_{t}} \mathbf{G}^{(1)}+\frac{2}{\delta_{t}}\left(\tilde{\mathbf{G}}^{(1)}-\hat{\mathbf{G}}^{(1)}\right), \\
\varepsilon^{2}: \partial_{t 2} \mathbf{m}^{(0)}+\left(\mathbf{I} \partial_{t 1}+\mathbf{D}_{1}\right) \tilde{\mathbf{G}}^{(1)}=-\frac{\mathbf{S}}{\delta_{t}} \mathbf{m}^{(2)}+\frac{\mathbf{S}}{\delta_{t}} \mathbf{Q}_{m}^{(2)}-\frac{\mathbf{R}}{\delta_{t}}\left(\mathbf{I}-\frac{\mathbf{S}}{2}\right) \mathbf{m}^{(2)},
\end{gathered}
$$

where the $\varepsilon^{1}$-order equation has been used to simplify the $\varepsilon^{2}$-order equation.

\section{A. Zeroth-order equation}

Based on the zeroth-order ( $\varepsilon^{0}$-order) equation [i.e., Eq. (9a)], we have

$$
\varepsilon^{0}: \mathbf{m}^{(0)}=\mathbf{m}^{\mathrm{eq}} .
$$

Thus, the corresponding equations for conserved moments $\left(m_{0}, m_{3}\right.$, and $\left.m_{5}\right)$ are

$$
\varepsilon^{0}:\left\{\begin{array}{l}
m_{0}^{(0)}=m_{0}^{\mathrm{eq}} \\
m_{3}^{(0)}=m_{3}^{\mathrm{eq}} \\
m_{5}^{(0)}=m_{5}^{\mathrm{eq}} .
\end{array}\right.
$$


By substituting the Chapman-Enskog expansions [i.e., Eq. (6a)] into the definitions $\rho=\sum_{i} f_{i}$ and $\rho \mathbf{u}=\sum_{i} \mathbf{e}_{i} f_{i}+$ $\delta_{t} \mathbf{F} / 2$, and considering Eq. (11), we can obtain

$$
\begin{cases}G_{0}^{(1)}=0, & m_{0}^{(n)}=0(\forall n \geq 2), \\ G_{3}^{(1)}=0, & m_{3}^{(n)}=0(\forall n \geq 2), \\ G_{5}^{(1)}=0, & m_{5}^{(n)}=0(\forall n \geq 2),\end{cases}
$$

which further indicate that $\tilde{G}_{0}^{(1)}=\hat{G}_{0}^{(1)}=0, \tilde{G}_{3}^{(1)}=\hat{G}_{3}^{(1)}=0$, and $\tilde{G}_{5}^{(1)}=\hat{G}_{5}^{(1)}=0$.

\section{B. First-order equation}

To deduce the macroscopic conservation equation, the equations for $m_{0}, m_{3}$, and $m_{5}$ are extracted from the firstorder $\left(\varepsilon^{1}\right.$-order) equation [i.e., Eq. (9b)] as follows

$$
\varepsilon^{1}:\left\{\begin{array}{l}
\partial_{t 1} m_{0}^{(0)}+c \partial_{x 1} m_{3}^{(0)}+c \partial_{y 1} m_{5}^{(0)}-F_{m, 0}^{(1)}=-\frac{s_{0}}{\delta_{t}} G_{0}^{(1)} \\
\partial_{t 1} m_{3}^{(0)}+c \partial_{x 1}\left(\frac{2}{3} m_{0}^{(0)}+\frac{1}{6} m_{1}^{(0)}+\frac{1}{2} m_{7}^{(0)}\right)+c \partial_{y 1} m_{8}^{(0)}-F_{m, 3}^{(1)}=-\frac{s_{j}}{\delta_{t}} G_{3}^{(1)} \\
\partial_{t 1} m_{5}^{(0)}+c \partial_{x 1} m_{8}^{(0)}+c \partial_{y 1}\left(\frac{2}{3} m_{0}^{(0)}+\frac{1}{6} m_{1}^{(0)}-\frac{1}{2} m_{7}^{(0)}\right)-F_{m, 5}^{(1)}=-\frac{s_{j}}{\delta_{t}} G_{5}^{(1)}
\end{array}\right.
$$

Considering Eqs. (10) and (12), Eq. (13) can be finally simplified as

$$
\varepsilon^{1}:\left\{\begin{array}{l}
\partial_{t 1} \rho+\nabla_{1} \cdot(\rho \mathbf{u})=0 \\
\partial_{t 1}(\rho \mathbf{u})+\nabla_{1} \cdot(\rho \mathbf{u u})=-\nabla_{1} p_{\mathrm{LBE}}+\mathbf{F}^{(1)}
\end{array}\right.
$$

where the recovered EOS $p_{\mathrm{LBE}}$ is

$$
p_{\mathrm{LBE}}=c_{s}^{2}\left[\left(2+\alpha_{1}\right) \rho+\beta_{1} \eta\right],
$$

and $c_{s}=c / \sqrt{3}$ is the lattice sound speed. Based on Eq. (14), the following relations for time derivative terms can be derived

$$
\begin{aligned}
& \partial_{t 1} \rho=-\nabla_{1} \cdot(\rho \mathbf{u}), \\
& \partial_{t 1}(\rho \mathbf{u})=-\nabla_{1} p_{\mathrm{LBE}}+\mathbf{F}^{(1)}-\nabla_{1} \cdot(\rho \mathbf{u u}), \\
& \partial_{t 1}(\rho \mathbf{u u})=-\left[\left(\nabla_{1} p_{\mathrm{LBE}}\right) \mathbf{u}\right]+\left[\mathbf{F}^{(1)} \mathbf{u}\right]-\nabla_{1} \cdot(\rho \mathbf{u u u}), \\
& \partial_{t 1}(\rho \mathbf{u u u})=-\left[\left(\nabla_{1} p_{\mathrm{LBE}}\right) \mathbf{u u}\right]+\left[\mathbf{F}^{(1)} \mathbf{u u}\right]-\nabla_{1} \cdot(\rho \mathbf{u u u u}), \\
& \partial_{t 1}(\rho \mathbf{u u u u})=-\left[\left(\nabla_{1} p_{\mathrm{LBE}}\right) \mathbf{u u u}\right]+\left[\mathbf{F}^{(1)} \mathbf{u u u}\right]-\nabla_{1} \cdot(\rho \mathbf{u u u u u}),
\end{aligned}
$$

in which $[\bullet]$ denotes permutation, e.g., $\left[\left(\nabla_{1} p_{\mathrm{LBE}}\right) \mathbf{u u}\right]=\left(\nabla_{1} p_{\mathrm{LBE}}\right) \mathbf{u u}+\mathbf{u}\left(\nabla_{1} p_{\mathrm{LBE}}\right) \mathbf{u}+\mathbf{u u}\left(\nabla_{1} p_{\mathrm{LBE}}\right)$ and $\left[\mathbf{F}^{(1)} \mathbf{u u}\right]=$ $\mathbf{F}^{(1)} \mathbf{u u}+\mathbf{u} \mathbf{F}^{(1)} \mathbf{u}+\mathbf{u} \mathbf{u} \mathbf{F}^{(1)}$. Here, we would like to point out that the last term $\nabla_{1} \cdot(\rho \mathbf{u u u})$ in the relation for $\partial_{t 1}(\rho \mathbf{u u})$ corresponds to the cubic terms of velocity in the recovered macroscopic equation at Navier-Stokes level for the classical LB model, which are usually ignored under the low Mach number condition.

\section{Second-order equation}

Extracting the equations for $m_{0}, m_{3}$, and $m_{5}$ from the second-order $\left(\varepsilon^{2}\right.$-order $)$ equation [i.e., Eq. (9c)], we have

$$
\varepsilon^{2}:\left\{\begin{array}{l}
\partial_{t 2} m_{0}^{(0)}+\partial_{t 1} \tilde{G}_{0}^{(1)}+c \partial_{x 1} \tilde{G}_{3}^{(1)}+c \partial_{y 1} \tilde{G}_{5}^{(1)}=-\frac{s_{0}}{\delta_{t}} m_{0}^{(2)} \\
\partial_{t 2} m_{3}^{(0)}+\partial_{t 1} \tilde{G}_{3}^{(1)}+c \partial_{x 1}\left(\frac{2}{3} \tilde{G}_{0}^{(1)}+\frac{1}{6} \tilde{G}_{1}^{(1)}+\frac{1}{2} \tilde{G}_{7}^{(1)}\right)+c \partial_{y 1} \tilde{G}_{8}^{(1)}=-\frac{s_{j}}{\delta_{t}} m_{3}^{(2)} \\
\partial_{t 2} m_{5}^{(0)}+\partial_{t 1} \tilde{G}_{5}^{(1)}+c \partial_{x 1} \tilde{G}_{8}^{(1)}+c \partial_{y 1}\left(\frac{2}{3} \tilde{G}_{0}^{(1)}+\frac{1}{6} \tilde{G}_{1}^{(1)}-\frac{1}{2} \tilde{G}_{7}^{(1)}\right)=-\frac{s_{j}}{\delta_{t}} m_{5}^{(2)}
\end{array}\right.
$$


With the help of Eqs. (10) and (12), Eq. (17) can be simplified as

$$
\varepsilon^{2}:\left\{\begin{array}{l}
\partial_{t 2} \rho=0 \\
\partial_{t 2}(\rho \mathbf{u})=\nabla_{1} \cdot \boldsymbol{\Pi}^{(1)}
\end{array}\right.
$$

where the viscous stress tensor $\boldsymbol{\Pi}^{(1)}$ is given as

$$
\boldsymbol{\Pi}^{(1)}=-c^{2}\left[\begin{array}{cc}
\frac{1}{2} \tilde{G}_{7}^{(1)} & \tilde{G}_{8}^{(1)} \\
\tilde{G}_{8}^{(1)} & -\frac{1}{2} \tilde{G}_{7}^{(1)}
\end{array}\right]-c^{2}\left[\begin{array}{cc}
\frac{1}{6} \tilde{G}_{1}^{(1)} & 0 \\
0 & \frac{1}{6} \tilde{G}_{1}^{(1)}
\end{array}\right] .
$$

\section{Newtonian viscous stress}

In order to calculate the viscous stress tensor given by Eq. (19), the equations for the related moments $\left(m_{1}, m_{7}\right.$, and $\left.m_{8}\right)$ at the order of $\varepsilon^{1}$ are extracted from Eq. (9b) as follows

$$
\begin{gathered}
-\frac{1}{\delta_{t}}\left(s_{e} G_{1}^{(1)}+k s_{\varepsilon} \omega_{e} G_{2}^{(1)}+h \hat{u}_{x} s_{q} \omega_{e} G_{4}^{(1)}+h \hat{u}_{y} s_{q} \omega_{e} G_{6}^{(1)}\right)+\frac{2}{\delta_{t}}\left(\tilde{G}_{1}^{(1)}-\hat{G}_{1}^{(1)}\right) \\
=\partial_{t 1} m_{1}^{(0)}+c \partial_{x 1}\left(m_{3}^{(0)}+m_{4}^{(0)}\right)+c \partial_{y 1}\left(m_{5}^{(0)}+m_{6}^{(0)}\right)-F_{m, 1}^{(1)}, \\
-\frac{1}{\delta_{t}}\left(s_{p} G_{7}^{(1)}+2 b \hat{u}_{x} s_{q} \omega_{p} G_{4}^{(1)}-2 b \hat{u}_{y} s_{q} \omega_{p} G_{6}^{(1)}\right)+\frac{2}{\delta_{t}}\left(\tilde{G}_{7}^{(1)}-\hat{G}_{7}^{(1)}\right) \\
=\partial_{t 1} m_{7}^{(0)}+c \partial_{x 1}\left(\frac{1}{3} m_{3}^{(0)}-\frac{1}{3} m_{4}^{(0)}\right)-c \partial_{y 1}\left(\frac{1}{3} m_{5}^{(0)}-\frac{1}{3} m_{6}^{(0)}\right)-F_{m, 7}^{(1)}, \\
-\frac{1}{\delta_{t}}\left(s_{p} G_{8}^{(1)}+b \hat{u}_{y} s_{q} \omega_{p} G_{4}^{(1)}+b \hat{u}_{x} s_{q} \omega_{p} G_{6}^{(1)}\right)+\frac{2}{\delta_{t}}\left(\tilde{G}_{8}^{(1)}-\hat{G}_{8}^{(1)}\right) \\
=\partial_{t 1} m_{8}^{(0)}+c \partial_{x 1}\left(\frac{2}{3} m_{5}^{(0)}+\frac{1}{3} m_{6}^{(0)}\right)+c \partial_{y 1}\left(\frac{2}{3} m_{3}^{(0)}+\frac{1}{3} m_{4}^{(0)}\right)-F_{m, 8}^{(1)},
\end{gathered}
$$

where the involved $\varepsilon^{1}$-order terms $G_{2}^{(1)}, G_{4}^{(1)}$, and $G_{6}^{(1)}$ can also be obtained from Eq. (9b)

$$
\begin{gathered}
-\frac{1}{\delta_{t}} s_{\varepsilon} G_{2}^{(1)}=\partial_{t 1} m_{2}^{(0)}+c \partial_{x 1} m_{4}^{(0)}+c \partial_{y 1} m_{6}^{(0)}-F_{m, 2}^{(1)}, \\
-\frac{1}{\delta_{t}} s_{q} G_{4}^{(1)}=\partial_{t 1} m_{4}^{(0)}+c \partial_{x 1}\left(\frac{1}{3} m_{1}^{(0)}+\frac{1}{3} m_{2}^{(0)}-m_{7}^{(0)}\right)+c \partial_{y 1} m_{8}^{(0)}-F_{m, 4}^{(1)}, \\
-\frac{1}{\delta_{t}} s_{q} G_{6}^{(1)}=\partial_{t 1} m_{6}^{(0)}+c \partial_{x 1} m_{8}^{(0)}+c \partial_{y 1}\left(\frac{1}{3} m_{1}^{(0)}+\frac{1}{3} m_{2}^{(0)}+m_{7}^{(0)}\right)-F_{m, 6}^{(1)} .
\end{gathered}
$$

Multiplying Eqs. (21a), (21b), and (21c) by $k, h \hat{u}_{x}$, and $h \hat{u}_{y}$, respectively, and then adding the results to Eq. (20a), we can obtain

$$
\begin{aligned}
-\frac{1}{\delta_{t}} \frac{2 s_{e}}{2-s_{e}} \hat{G}_{1}^{(1)}+\frac{2}{\delta_{t}}\left(\tilde{G}_{1}^{(1)}-\hat{G}_{1}^{(1)}\right)= & \partial_{t 1} m_{1}^{(0)}+c \partial_{x 1}\left(m_{3}^{(0)}+m_{4}^{(0)}\right)+c \partial_{y 1}\left(m_{5}^{(0)}+m_{6}^{(0)}\right)-F_{m, 1}^{(1)} \\
& +k\left[\partial_{t 1} m_{2}^{(0)}+c \partial_{x 1} m_{4}^{(0)}+c \partial_{y 1} m_{6}^{(0)}-F_{m, 2}^{(1)}\right] \\
& +h \hat{u}_{x}\left[\partial_{t 1} m_{4}^{(0)}+c \partial_{x 1}\left(\frac{1}{3} m_{1}^{(0)}+\frac{1}{3} m_{2}^{(0)}-m_{7}^{(0)}\right)+c \partial_{y 1} m_{8}^{(0)}-F_{m, 4}^{(1)}\right] \\
& +h \hat{u}_{y}\left[\partial_{t 1} m_{6}^{(0)}+c \partial_{x 1} m_{8}^{(0)}+c \partial_{y 1}\left(\frac{1}{3} m_{1}^{(0)}+\frac{1}{3} m_{2}^{(0)}+m_{7}^{(0)}\right)-F_{m, 6}^{(1)}\right]
\end{aligned}
$$

With the help of Eqs. (10) and (16), as well as $\mathbf{m}^{\mathrm{eq}}$ and $\mathbf{F}_{m}$, Eq. (22) can be simplified after lengthy algebra as follows

$$
\begin{aligned}
-\frac{1}{\delta_{t}} \frac{2 s_{e}}{2-s_{e}} \hat{G}_{1}^{(1)}+\frac{2}{\delta_{t}}\left(\tilde{G}_{1}^{(1)}-\hat{G}_{1}^{(1)}\right)= & -\left(2 \alpha_{1}+k \alpha_{2}+k\right) \nabla_{1} \cdot(\rho \mathbf{u})+\frac{h\left[\left(\alpha_{2}-4\right) \beta_{1}-\left(\alpha_{1}+2\right) \beta_{2}\right]}{3 \beta_{1}} \mathbf{u} \cdot \nabla_{1} \rho \\
& +\frac{h\left(3 \beta_{1}+\beta_{2}\right)-6 \beta_{1}(1-k)}{\beta_{1}} \mathbf{u} \cdot \nabla_{1} \hat{p}_{\mathrm{LBE}}+\left(2 \beta_{1}+k \beta_{2}\right) \partial_{t 1} \eta \\
& -c\left\{9(2 k+h) \hat{u}_{x} \hat{u}_{y}^{2} \partial_{x 1} \hat{p}_{\mathrm{LBE}}+9(2 k+h) \hat{u}_{x}^{2} \hat{u}_{y} \partial_{y 1} \hat{p}_{\mathrm{LBE}}\right\} \\
& -c\left\{3\left[(1-k) \hat{u}_{x}^{3}-(2 k+h) \hat{u}_{x} \hat{u}_{y}^{2}\right] \partial_{x 1} \rho+3\left[(1-k) \hat{u}_{y}^{3}-(2 k+h) \hat{u}_{x}^{2} \hat{u}_{y}\right] \partial_{y 1} \rho\right\} \\
& -c\left\{\left[9(1-k) \hat{u}_{x}^{2}-2(3 k+h) \hat{u}_{y}^{2}\right] \rho \partial_{x 1} \hat{u}_{x}+\left[9(1-k) \hat{u}_{y}^{2}-2(3 k+h) \hat{u}_{x}^{2}\right] \rho \partial_{y 1} \hat{u}_{y}\right\} \\
\left.-4(3 k+h) \hat{u}_{x} \hat{u}_{y} \rho\left(\partial_{x 1} \hat{u}_{y}+\partial_{y 1} \hat{u}_{x}\right)\right\} & -c\left\{9 k \nabla_{1} \cdot\left(\rho \hat{\mathbf{u}} \hat{u}_{x}^{2} \hat{u}_{y}^{2}\right)+3 h \hat{u}_{y} \partial_{x 1}\left(\rho \hat{u}_{x}^{3} \hat{u}_{y}\right)+3 h \hat{u}_{x} \partial_{y 1}\left(\rho \hat{u}_{x} \hat{u}_{y}^{3}\right)\right\},
\end{aligned}
$$


where $\hat{p}_{\mathrm{LBE}}=p_{\mathrm{LBE}} / c^{2}$. In order to correctly recover the Newtonian viscous stress, one should have

$$
\left\{\begin{array}{l}
2 \alpha_{1}+k \alpha_{2}+k=\frac{h\left[\left(\alpha_{2}-4\right) \beta_{1}-\left(\alpha_{1}+2\right) \beta_{2}\right]}{3 \beta_{1}} \equiv-2 \varpi, \\
\frac{h\left(3 \beta_{1}+\beta_{2}\right)-6 \beta_{1}(1-k)}{\beta_{1}}=0, \\
2 \beta_{1}+k \beta_{2}=0 .
\end{array}\right.
$$

Here, a crucial parameter $\varpi$ is further introduced, and it is worth emphasizing that $\varpi$ cannot be simply set to 1 following the classical LB model with ideal-gas EOS. Otherwise, singularity will be encountered [see Eq. (31)] and the present model should degenerate into the classical LB model. Moreover, there exist some third- and fifth-order terms of velocity in Eq. (23), which can be simply ignored under the low Mach number condition. As a better choice, eliminating the third-order terms will be discussed in next subsection.

Multiplying Eqs. (21b) and (21c) by $2 b \hat{u}_{x}$ and $-2 b \hat{u}_{y}$, respectively, and then adding the results to Eq. (20b), we can obtain

$$
\begin{aligned}
-\frac{1}{\delta_{t}} \frac{2 s_{p}}{2-s_{p}} \hat{G}_{7}^{(1)}+\frac{2}{\delta_{t}}\left(\tilde{G}_{7}^{(1)}-\hat{G}_{7}^{(1)}\right)= & \partial_{t 1} m_{7}^{(0)}+c \partial_{x 1}\left(\frac{1}{3} m_{3}^{(0)}-\frac{1}{3} m_{4}^{(0)}\right)-c \partial_{y 1}\left(\frac{1}{3} m_{5}^{(0)}-\frac{1}{3} m_{6}^{(0)}\right)-F_{m, 7}^{(1)} \\
& +2 b \hat{u}_{x}\left[\partial_{t 1} m_{4}^{(0)}+c \partial_{x 1}\left(\frac{1}{3} m_{1}^{(0)}+\frac{1}{3} m_{2}^{(0)}-m_{7}^{(0)}\right)+c \partial_{y 1} m_{8}^{(0)}-F_{m, 4}^{(1)}\right] \\
& -2 b \hat{u}_{y}\left[\partial_{t 1} m_{6}^{(0)}+c \partial_{x 1} m_{8}^{(0)}+c \partial_{y 1}\left(\frac{1}{3} m_{1}^{(0)}+\frac{1}{3} m_{2}^{(0)}+m_{7}^{(0)}\right)-F_{m, 6}^{(1)}\right] .
\end{aligned}
$$

By utilizing Eqs. (10) and (16), as well as $\mathbf{m}^{\mathrm{eq}}$ and $\mathbf{F}_{m}$, Eq. (25) can be simplified after lengthy algebra as follows

$$
\begin{aligned}
-\frac{1}{\delta_{t}} \frac{2 s_{p}}{2-s_{p}} \hat{G}_{7}^{(1)}+\frac{2}{\delta_{t}}\left(\tilde{G}_{7}^{(1)}-\hat{G}_{7}^{(1)}\right)= & \frac{2}{3}\left[\partial_{x 1}\left(\rho u_{x}\right)-\partial_{y 1}\left(\rho u_{y}\right)\right]+\frac{2}{3} \frac{b\left[\left(\alpha_{2}-4\right) \beta_{1}-\left(\alpha_{1}+2\right) \beta_{2}\right]}{\beta_{1}}\left(u_{x} \partial_{x 1} \rho-u_{y} \partial_{y 1} \rho\right) \\
& +\frac{(6 b-2) \beta_{1}+2 b \beta_{2}}{\beta_{1}}\left(u_{x} \partial_{x 1} \hat{p}_{\mathrm{LBE}}-u_{y} \partial_{y 1} \hat{p}_{\mathrm{LBE}}\right) \\
& +c\left\{6 b \hat{u}_{x} \hat{u}_{y}^{2} \partial_{x 1} \hat{p}_{\mathrm{LBE}}-6 b \hat{u}_{x}^{2} \hat{u}_{y} \partial_{y 1} \hat{p}_{\mathrm{LBE}}\right\} \\
& -c\left\{\left(\hat{u}_{x}^{3}+2 b \hat{u}_{x} \hat{u}_{y}^{2}\right) \partial_{x 1} \rho-\left(\hat{u}_{y}^{3}+2 b \hat{u}_{x}^{2} \hat{u}_{y}\right) \partial_{y 1} \rho\right\} \\
& -c\left\{\left(3 \hat{u}_{x}^{2}+4 b \hat{u}_{y}^{2}\right) \rho \partial_{x 1} \hat{u}_{x}-\left(3 \hat{u}_{y}^{2}+4 b \hat{u}_{x}^{2}\right) \rho \partial_{y 1} \hat{u}_{y}\right\} \\
& +c\left\{6 b \hat{u}_{y} \partial_{x 1}\left(\rho \hat{u}_{x}^{3} \hat{u}_{y}\right)-6 b \hat{u}_{x} \partial_{y 1}\left(\rho \hat{u}_{x} \hat{u}_{y}^{3}\right)\right\} .
\end{aligned}
$$

In order to correctly recover the Newtonian viscous stress, one should have

$$
\left\{\begin{array}{l}
\frac{b\left[\left(\alpha_{2}-4\right) \beta_{1}-\left(\alpha_{1}+2\right) \beta_{2}\right]}{\beta_{1}}=-1, \\
\frac{(6 b-2) \beta_{1}+2 b \beta_{2}}{\beta_{1}}=0 .
\end{array}\right.
$$

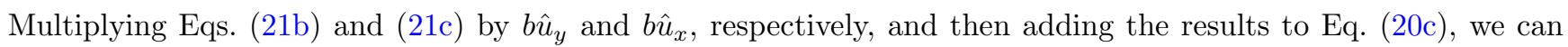
obtain

$$
\begin{aligned}
-\frac{1}{\delta_{t}} \frac{2 s_{p}}{2-s_{p}} \hat{G}_{8}^{(1)}+\frac{2}{\delta_{t}}\left(\tilde{G}_{8}^{(1)}-\hat{G}_{8}^{(1)}\right)= & \partial_{t 1} m_{8}^{(0)}+c \partial_{x 1}\left(\frac{2}{3} m_{5}^{(0)}+\frac{1}{3} m_{6}^{(0)}\right)+c \partial_{y 1}\left(\frac{2}{3} m_{3}^{(0)}+\frac{1}{3} m_{4}^{(0)}\right)-F_{m, 8}^{(1)} \\
& +b \hat{u}_{y}\left[\partial_{t 1} m_{4}^{(0)}+c \partial_{x 1}\left(\frac{1}{3} m_{1}^{(0)}+\frac{1}{3} m_{2}^{(0)}-m_{7}^{(0)}\right)+c \partial_{y 1} m_{8}^{(0)}-F_{m, 4}^{(1)}\right] \\
& +b \hat{u}_{x}\left[\partial_{t 1} m_{6}^{(0)}+c \partial_{x 1} m_{8}^{(0)}+c \partial_{y 1}\left(\frac{1}{3} m_{1}^{(0)}+\frac{1}{3} m_{2}^{(0)}+m_{7}^{(0)}\right)-F_{m, 6}^{(1)}\right] .
\end{aligned}
$$

With the help of Eqs. (10) and (16), as well as $\mathbf{m}^{\mathrm{eq}}$ and $\mathbf{F}_{m}$, Eq. (28) can be simplified as follows

$$
\begin{aligned}
-\frac{1}{\delta_{t}} \frac{2 s_{p}}{2-s_{p}} \hat{G}_{8}^{(1)}+\frac{2}{\delta_{t}}\left(\tilde{G}_{8}^{(1)}-\hat{G}_{8}^{(1)}\right)= & \frac{1}{3}\left[\partial_{x 1}\left(\rho u_{y}\right)+\partial_{y 1}\left(\rho u_{x}\right)\right]+\frac{1}{3} \frac{b\left[\left(\alpha_{2}-4\right) \beta_{1}-\left(\alpha_{1}+2\right) \beta_{2}\right]}{\beta_{1}}\left(u_{y} \partial_{x 1} \rho+u_{x} \partial_{y 1} \rho\right) \\
& +\frac{(3 b-1) \beta_{1}+b \beta_{2}}{\beta_{1}}\left(u_{y} \partial_{x 1} \hat{p}_{\mathrm{LBE}}+u_{x} \partial_{y 1} \hat{p}_{\mathrm{LBE}}\right) \\
& -c\left\{3 b\left(\hat{u}_{y}^{3}+2 \hat{u}_{x}^{2} \hat{u}_{y}\right) \partial_{x 1} \hat{p}_{\mathrm{LBE}}+3 b\left(\hat{u}_{x}^{3}+2 \hat{u}_{x} \hat{u}_{y}^{2}\right) \partial_{y 1} \hat{p}_{\mathrm{LBE}}\right\} \\
& +c\left\{b\left(\hat{u}_{y}^{3}+2 \hat{u}_{x}^{2} \hat{u}_{y}\right) \partial_{x 1} \rho+b\left(\hat{u}_{x}^{3}+2 \hat{u}_{x} \hat{u}_{y}^{2}\right) \partial_{y 1} \rho\right\} \\
& +c\left\{2 b \hat{u}_{x} \hat{u}_{y} \rho \partial_{x 1} \hat{u}_{x}+2 b \hat{u}_{x} \hat{u}_{y} \rho \partial_{y 1} \hat{u}_{y}+2 b\left(\hat{u}_{x}^{2}+\hat{u}_{y}^{2}\right) \rho\left(\partial_{x 1} \hat{u}_{y}+\partial_{y 1} \hat{u}_{x}\right)\right\} \\
& -c\left\{3 b \hat{u}_{x} \partial_{x 1}\left(\rho \hat{u}_{x}^{3} \hat{u}_{y}\right)+3 b \hat{u}_{y} \partial_{y 1}\left(\rho \hat{u}_{x} \hat{u}_{y}^{3}\right)\right\} .
\end{aligned}
$$


Similarly, in order to correctly recover the Newtonian viscous stress, one should have

$$
\left\{\begin{array}{l}
\frac{b\left[\left(\alpha_{2}-4\right) \beta_{1}-\left(\alpha_{1}+2\right) \beta_{2}\right]}{\beta_{1}}=-1, \\
\frac{(3 b-1) \beta_{1}+b \beta_{2}}{\beta_{1}}=0 .
\end{array}\right.
$$

Here, we note that Eq. (30) is actually the same as Eq. (27), which indicates that the diagonal and non-diagonal elements in the traceless part of viscous stress tensor can be correctly recovered, or not, at the same time.

Eqs. (24), (27), and (30) give the constraints on the coefficients in $\mathbf{~ m}^{\mathrm{eq}}$ and $\mathbf{S}$ required for the correct recovery of Newtonian viscous stress. Considering the classical LB model can be viewed as a special case of the present LB model, Eqs. (24), (27), and (30) must be compatible with each other. Solving these equations, we have

$$
\alpha_{2}=-\frac{2 \alpha_{1}+\varpi+1}{1-\varpi}, \quad \beta_{2}=-\frac{2 \beta_{1}}{1-\varpi}, \quad k=1-\varpi, \quad h=\frac{6 \varpi(1-\varpi)}{1-3 \varpi}, \quad b=\frac{1-\varpi}{1-3 \varpi} .
$$

In practical applications, $\alpha_{1}$ is set to -1 according to the ordinary equilibrium moment function derived from the Maxwell-Boltzmann distribution, and $\beta_{1}$ is set to 1 as ordinary. Therefore, all the coefficients $\alpha_{2}, \beta_{2}, k, h$, and $b$ are uniquely determined by $\varpi$.

\section{Cubic terms of velocity}

From Eqs. (23), (26), and (29), and considering $\mathbf{R} \sim O\left(M a^{2}\right), \mathbf{T} \sim O\left(M a^{3}\right)$, and $\mathbf{X} \sim O\left(M a^{3}\right)$, we have $\hat{G}_{1}^{(1)} \sim$ $O(M a), \hat{G}_{7}^{(1)} \sim O(M a)$, and $\hat{G}_{8}^{(1)} \sim O(M a)$. Thus, the leading-order terms of Eqs. (23), (26), and (29) are given as

$$
\left\{\begin{array}{l}
-\frac{1}{\delta_{t}} \frac{2 s_{e}}{2-s_{e}} \hat{G}_{1}^{(1)}=2 \varpi \rho\left(\partial_{x 1} u_{x}+\partial_{y 1} u_{y}\right)+O\left(M a^{3}\right), \\
-\frac{1}{\delta_{t}} \frac{2 s_{p}}{2-s_{p}} \hat{G}_{7}^{(1)}=\frac{2}{3} \rho\left(\partial_{x 1} u_{x}-\partial_{y 1} u_{y}\right)+O\left(M a^{3}\right), \\
-\frac{1}{\delta_{t}} \frac{2 s_{p}}{2-s_{p}} \hat{G}_{8}^{(1)}=\frac{1}{3} \rho\left(\partial_{x 1} u_{y}+\partial_{y 1} u_{x}\right)+O\left(M a^{3}\right) .
\end{array}\right.
$$

Based on Eq. (23) and $\tilde{G}_{1}^{(1)}=\hat{G}_{1}^{(1)}-\frac{1}{2}\left(R_{11} \hat{G}_{1}^{(1)}+R_{17} \hat{G}_{7}^{(1)}+R_{18} \hat{G}_{8}^{(1)}+\delta_{x} \mathbf{T}_{1} \cdot \nabla_{1} \rho+\delta_{x} \mathbf{X}_{1} \cdot \nabla_{1} \hat{p}_{\mathrm{LBE}}\right)$, we have

$$
\begin{aligned}
-\frac{1}{\delta_{t}} \frac{2 s_{e}}{2-s_{e}} \tilde{G}_{1}^{(1)}= & 2 \varpi \rho\left(\partial_{x 1} u_{x}+\partial_{y 1} u_{y}\right)+\frac{1}{\delta_{t}} \frac{2}{2-s_{e}}\left(R_{11} \hat{G}_{1}^{(1)}+R_{17} \hat{G}_{7}^{(1)}+R_{18} \hat{G}_{8}^{(1)}+\delta_{x} \mathbf{T}_{1} \cdot \nabla_{1} \rho+\delta_{x} \mathbf{X}_{1} \cdot \nabla_{1} \hat{p}_{\mathrm{LBE}}\right) \\
& -c\left\{9(2 k+h) \hat{u}_{x} \hat{u}_{y}^{2} \partial_{x 1} \hat{p}_{\mathrm{LBE}}+9(2 k+h) \hat{u}_{x}^{2} \hat{u}_{y} \partial_{y 1} \hat{p}_{\mathrm{LBE}}\right\} \\
& -c\left\{3\left[(1-k) \hat{u}_{x}^{3}-(2 k+h) \hat{u}_{x} \hat{u}_{y}^{2}\right] \partial_{x 1} \rho+3\left[(1-k) \hat{u}_{y}^{3}-(2 k+h) \hat{u}_{x}^{2} \hat{u}_{y}\right] \partial_{y 1} \rho\right\} \\
& -c\left\{\left[9(1-k) \hat{u}_{x}^{2}-2(3 k+h) \hat{u}_{y}^{2}\right] \rho \partial_{x 1} \hat{u}_{x}+\left[9(1-k) \hat{u}_{y}^{2}-2(3 k+h) \hat{u}_{x}^{2}\right] \rho \partial_{y 1} \hat{u}_{y}\right\} \\
& \left.-4(3 k+h) \hat{u}_{x} \hat{u}_{y} \rho\left(\partial_{x 1} \hat{u}_{y}+\partial_{y 1} \hat{u}_{x}\right)\right\} \\
& -c\left\{9 k \nabla_{1} \cdot\left(\rho \hat{\mathbf{u}} \hat{u}_{x}^{2} \hat{u}_{y}^{2}\right)+3 h \hat{u}_{y} \partial_{x 1}\left(\rho \hat{u}_{x}^{3} \hat{u}_{y}\right)+3 h \hat{u}_{x} \partial_{y 1}\left(\rho \hat{u}_{x} \hat{u}_{y}^{3}\right)\right\} .
\end{aligned}
$$

In order to eliminate the cubic terms of velocity in Eq. (33) and with the consideration of Eq. (32), we can set

$$
\begin{gathered}
R_{11}=-\frac{s_{e}}{4 \varpi}[9(1-k)-2(3 k+h)]\left(\hat{u}_{x}^{2}+\hat{u}_{y}^{2}\right), \quad R_{17}=-\frac{3 s_{p}\left(2-s_{e}\right)}{4\left(2-s_{p}\right)}[9(1-k)+2(3 k+h)]\left(\hat{u}_{x}^{2}-\hat{u}_{y}^{2}\right), \\
R_{18}=\frac{12 s_{p}\left(2-s_{e}\right)}{2-s_{p}}(3 k+h) \hat{u}_{x} \hat{u}_{y}, \quad \mathbf{T}_{1}=\frac{3\left(2-s_{e}\right)}{2}\left[\begin{array}{l}
(1-k) \hat{u}_{x}^{3}-(2 k+h) \hat{u}_{x} \hat{u}_{y}^{2} \\
(1-k) \hat{u}_{y}^{3}-(2 k+h) \hat{u}_{x}^{2} \hat{u}_{y}
\end{array}\right], \quad \mathbf{X}_{1}=\frac{9\left(2-s_{e}\right)}{2}(2 k+h)\left[\begin{array}{l}
\hat{u}_{x} \hat{u}_{y}^{2} \\
\hat{u}_{x}^{2} \hat{u}_{y}
\end{array}\right] .
\end{gathered}
$$

Thus, Eq. (33) can be finally simplified as

$$
-\frac{1}{\delta_{t}} \frac{2 s_{e}}{2-s_{e}} \tilde{G}_{1}^{(1)}=2 \varpi \rho\left(\partial_{x 1} u_{x}+\partial_{y 1} u_{y}\right)+O\left(M a^{5}\right) .
$$


From Eq. (26), and considering $\tilde{G}_{7}^{(1)}=\hat{G}_{7}^{(1)}-\frac{1}{2}\left(R_{71} \hat{G}_{1}^{(1)}+R_{77} \hat{G}_{7}^{(1)}+R_{78} \hat{G}_{8}^{(1)}+\delta_{x} \mathbf{T}_{7} \cdot \nabla_{1} \rho+\delta_{x} \mathbf{X}_{7} \cdot \nabla_{1} \hat{p}_{\mathrm{LBE}}\right)$, we have

$$
\begin{aligned}
-\frac{1}{\delta_{t}} \frac{2 s_{p}}{2-s_{p}} \tilde{G}_{7}^{(1)}= & \frac{2}{3} \rho\left(\partial_{x 1} u_{x}-\partial_{y 1} u_{y}\right)+\frac{1}{\delta_{t}} \frac{2}{2-s_{p}}\left(R_{71} \hat{G}_{1}^{(1)}+R_{77} \hat{G}_{7}^{(1)}+R_{78} \hat{G}_{8}^{(1)}+\delta_{x} \mathbf{T}_{7} \cdot \nabla_{1} \rho+\delta_{x} \mathbf{X}_{7} \cdot \nabla_{1} \hat{p}_{\mathrm{LBE}}\right) \\
& +c\left\{6 b \hat{u}_{x} \hat{u}_{y}^{2} \partial_{x 1} \hat{p}_{\mathrm{LBE}}-6 b \hat{u}_{x}^{2} \hat{u}_{y} \partial_{y 1} \hat{p}_{\mathrm{LBE}}\right\}-c\left\{\left(\hat{u}_{x}^{3}+2 b \hat{u}_{x} \hat{u}_{y}^{2}\right) \partial_{x 1} \rho-\left(\hat{u}_{y}^{3}+2 b \hat{u}_{x}^{2} \hat{u}_{y}\right) \partial_{y 1} \rho\right\} \\
& -c\left\{\left(3 \hat{u}_{x}^{2}+4 b \hat{u}_{y}^{2}\right) \rho \partial_{x 1} \hat{u}_{x}-\left(3 \hat{u}_{y}^{2}+4 b \hat{u}_{x}^{2}\right) \rho \partial_{y 1} \hat{u}_{y}\right\}+c\left\{6 b \hat{u}_{y} \partial_{x 1}\left(\rho \hat{u}_{x}^{3} \hat{u}_{y}\right)-6 b \hat{u}_{x} \partial_{y 1}\left(\rho \hat{u}_{x} \hat{u}_{y}^{3}\right)\right\} .
\end{aligned}
$$

To eliminate the additional cubic terms of velocity, and with the consideration of Eq. (32), we can set

$$
\begin{aligned}
& R_{71}=-\frac{s_{e}\left(2-s_{p}\right)}{4 \varpi\left(2-s_{e}\right)}(3-4 b)\left(\hat{u}_{x}^{2}-\hat{u}_{y}^{2}\right), \quad R_{77}=-\frac{3 s_{p}}{4}(3+4 b)\left(\hat{u}_{x}^{2}+\hat{u}_{y}^{2}\right), \\
& R_{78}=0, \quad \mathbf{T}_{7}=\frac{2-s_{p}}{2}\left[\begin{array}{c}
\hat{u}_{x}^{3}+2 b \hat{u}_{x} \hat{u}_{y}^{2} \\
-\hat{u}_{y}^{3}-2 b \hat{u}_{x}^{2} \hat{u}_{y}
\end{array}\right], \quad \mathbf{X}_{7}=-3\left(2-s_{p}\right) b\left[\begin{array}{c}
\hat{u}_{x} \hat{u}_{y}^{2} \\
-\hat{u}_{x}^{2} \hat{u}_{y}
\end{array}\right] .
\end{aligned}
$$

Thus, Eq. (36) is finally simplified as

$$
-\frac{1}{\delta_{t}} \frac{2 s_{p}}{2-s_{p}} \tilde{G}_{7}^{(1)}=\frac{2}{3} \rho\left(\partial_{x 1} u_{x}-\partial_{y 1} u_{y}\right)+O\left(M a^{5}\right) .
$$

Based on Eq. (29), and considering $\tilde{G}_{8}^{(1)}=\hat{G}_{8}^{(1)}-\frac{1}{2}\left(R_{81} \hat{G}_{1}^{(1)}+R_{87} \hat{G}_{7}^{(1)}+R_{88} \hat{G}_{8}^{(1)}+\delta_{x} \mathbf{T}_{8} \cdot \nabla_{1} \rho+\delta_{x} \mathbf{X}_{8} \cdot \nabla_{1} \hat{p}_{\mathrm{LBE}}\right)$, we have

$$
\begin{aligned}
-\frac{1}{\delta_{t}} \frac{2 s_{p}}{2-s_{p}} \tilde{G}_{8}^{(1)}= & \frac{1}{3} \rho\left(\partial_{x 1} u_{y}+\partial_{y 1} u_{x}\right)+\frac{1}{\delta_{t}} \frac{2}{2-s_{p}}\left(R_{81} \hat{G}_{1}^{(1)}+R_{87} \hat{G}_{7}^{(1)}+R_{88} \hat{G}_{8}^{(1)}+\delta_{x} \mathbf{T}_{8} \cdot \nabla_{1} \rho+\delta_{x} \mathbf{X}_{8} \cdot \nabla_{1} \hat{p}_{\mathrm{LBE}}\right) \\
& -c\left\{3 b\left(\hat{u}_{y}^{3}+2 \hat{u}_{x}^{2} \hat{u}_{y}\right) \partial_{x 1} \hat{p}_{\mathrm{LBE}}+3 b\left(\hat{u}_{x}^{3}+2 \hat{u}_{x} \hat{u}_{y}^{2}\right) \partial_{y 1} \hat{p}_{\mathrm{LBE}}\right\} \\
& +c\left\{b\left(\hat{u}_{y}^{3}+2 \hat{u}_{x}^{2} \hat{u}_{y}\right) \partial_{x 1} \rho+b\left(\hat{u}_{x}^{3}+2 \hat{u}_{x} \hat{u}_{y}^{2}\right) \partial_{y 1} \rho\right\} \\
& +c\left\{2 b \hat{u}_{x} \hat{u}_{y} \rho \partial_{x 1} \hat{u}_{x}+2 b \hat{u}_{x} \hat{u}_{y} \rho \partial_{y 1} \hat{u}_{y}+2 b\left(\hat{u}_{x}^{2}+\hat{u}_{y}^{2}\right) \rho\left(\partial_{x 1} \hat{u}_{y}+\partial_{y 1} \hat{u}_{x}\right)\right\} \\
& -c\left\{3 b \hat{u}_{x} \partial_{x 1}\left(\rho \hat{u}_{x}^{3} \hat{u}_{y}\right)+3 b \hat{u}_{y} \partial_{y 1}\left(\rho \hat{u}_{x} \hat{u}_{y}^{3}\right)\right\} .
\end{aligned}
$$

Similarly, to eliminate the additional cubic terms, we can set

$$
\begin{gathered}
R_{81}=\frac{s_{e}\left(2-s_{p}\right)}{\varpi\left(2-s_{e}\right)} b \hat{u}_{x} \hat{u}_{y}, \quad R_{87}=0, \\
R_{88}=6 s_{p} b\left(\hat{u}_{x}^{2}+\hat{u}_{y}^{2}\right), \quad \mathbf{T}_{8}=-\frac{2-s_{p}}{2} b\left[\begin{array}{l}
\hat{u}_{y}^{3}+2 \hat{u}_{x}^{2} \hat{u}_{y} \\
\hat{u}_{x}^{3}+2 \hat{u}_{x} \hat{u}_{y}^{2}
\end{array}\right], \quad \mathbf{X}_{8}=\frac{3\left(2-s_{p}\right)}{2} b\left[\begin{array}{l}
\hat{u}_{y}^{3}+2 \hat{u}_{x}^{2} \hat{u}_{y} \\
\hat{u}_{x}^{3}+2 \hat{u}_{x} \hat{u}_{y}^{2}
\end{array}\right],
\end{gathered}
$$

and then Eq. (39) is simplified as

$$
-\frac{1}{\delta_{t}} \frac{2 s_{p}}{2-s_{p}} \tilde{G}_{8}^{(1)}=\frac{1}{3} \rho\left(\partial_{x 1} u_{y}+\partial_{y 1} u_{x}\right)+O\left(M a^{5}\right) .
$$

As given by Eqs. (34), (37), and (40), the nonzero elements in $\mathbf{R}, \mathbf{T}$, and $\mathbf{X}$ can be uniquely and locally determined, and the results are also consistent with the aforementioned conditions $\mathbf{R} \sim O\left(M a^{2}\right), \mathbf{T} \sim O\left(M a^{3}\right)$, and $\mathbf{X} \sim O\left(M a^{3}\right)$. Based on Eqs. (35), (38), and (41), the viscous stress tensor given by Eq. (19) can be simplified as

$$
\boldsymbol{\Pi}^{(1)}=\rho \nu\left[\nabla_{1} \mathbf{u}+\mathbf{u} \nabla_{1}-\left(\nabla_{1} \cdot \mathbf{u}\right) \mathbf{I}\right]+\rho \varsigma\left(\nabla_{1} \cdot \mathbf{u}\right) \mathbf{I}+O\left(M a^{5}\right),
$$

where the kinematic viscosity $\nu$ and bulk viscosity $\varsigma$ are given as

$$
\nu=c_{s}^{2}\left(s_{p}^{-1}-\frac{1}{2}\right) \delta_{t}, \quad \varsigma=\varpi c_{s}^{2}\left(s_{e}^{-1}-\frac{1}{2}\right) \delta_{t} .
$$

\section{Macroscopic equation}

Combining the $\varepsilon^{1}$ - and $\varepsilon^{2}$-order equations [i.e., Eqs. (14) and (18)] and utilizing Eq. (42), the macroscopic equation at the Navier-Stokes level can be finally recovered as follows

$$
\left\{\begin{array}{l}
\partial_{t} \rho+\nabla \cdot(\rho \mathbf{u})=0, \\
\partial_{t}(\rho \mathbf{u})+\nabla \cdot(\rho \mathbf{u u})=-\nabla p_{\mathrm{LBE}}+\mathbf{F}+\nabla \cdot\{\rho \nu[\nabla \mathbf{u}+\mathbf{u} \nabla-(\nabla \cdot \mathbf{u}) \mathbf{I}]+\rho \varsigma(\nabla \cdot \mathbf{u}) \mathbf{I}\}+O\left(M a^{5}\right) .
\end{array}\right.
$$


Obviously, the Newtonian viscous stress is correctly recovered and thus the Galilean invariance can be satisfied. From the above second-order analysis, we can see that the force term $\mathbf{F}$ can be recovered with no discrete lattice effect at the $\varepsilon^{2}$-order, and the term $\mathbf{S Q}_{m}$ in $\mathrm{LB}$ equation for $\varepsilon^{3}$-order additional term makes no difference to the recovered macroscopic equation at the $\varepsilon^{2}$-order.

\section{THIRD-ORDER ANALYSIS}

The target of the present third-order analysis is to identify the leading-order terms at the $\varepsilon^{3}$-order, which should be seriously considered for multiphase flows [4]. These leading-order terms are mainly related to the density gradient in the interfacial region caused by the pairwise interaction force, which means that such terms are irrelevant to time and velocity. Therefore, a steady and stationary situation can be considered, which is simple but effective, and can also avoid leading to the Burnett level equation that is unnecessary and undesirable. In a steady and stationary situation, the $\varepsilon^{0}-, \varepsilon^{1}-, \varepsilon^{2}$, and $\varepsilon^{3}$-order equations in Eq. (7) can be simplified as

$$
\begin{gathered}
\varepsilon^{0}: \mathbf{m}^{(0)}=\mathbf{m}^{\mathrm{eq}} \\
\varepsilon^{1}: \partial_{t 1} \mathbf{m}^{(0)}+\mathbf{D}_{1} \mathbf{m}^{(0)}-\mathbf{F}_{m}^{(1)}=-\frac{\mathbf{S}}{\delta_{t}}\left(\mathbf{m}^{(1)}+\frac{\delta_{t}}{2} \mathbf{F}_{m}^{(1)}\right) \\
\varepsilon^{2}: \partial_{t 2} \mathbf{m}^{(0)}-\delta_{t} \mathbf{D}_{1}\left(\mathbf{S}^{-1}-\frac{\mathbf{I}}{2}\right)\left(\mathbf{D}_{1} \mathbf{m}^{(0)}-\mathbf{F}_{m}^{(1)}\right)=-\frac{\mathbf{S}}{\delta_{t}} \mathbf{m}^{(2)}+\frac{\mathbf{S}}{\delta_{t}} \mathbf{Q}_{m}^{(2)}, \\
\varepsilon^{3}: \partial_{t 3} \mathbf{m}^{(0)}+\delta_{t}^{2}\left[\mathbf{D}_{1}\left(\mathbf{S}^{-1}-\frac{\mathbf{I}}{2}\right) \mathbf{D}_{1}\left(\mathbf{S}^{-1}-\frac{\mathbf{I}}{2}\right)\left(\mathbf{D}_{1} \mathbf{m}^{(0)}-\mathbf{F}_{m}^{(1)}\right)-\frac{1}{12} \mathbf{D}_{1}^{3} \mathbf{m}^{(0)}\right]+\mathbf{D}_{1} \mathbf{Q}_{m}^{(2)}=-\frac{\mathbf{S}}{\delta_{t}} \mathbf{m}^{(3)},
\end{gathered}
$$

where the terms $\partial_{t 1} \mathbf{m}^{(0)}, \partial_{t 2} \mathbf{m}^{(0)}$, and $\partial_{t 3} \mathbf{m}^{(0)}$ are reserved as a gauge for the equations at different orders. Here, it is worth pointing out that the present improved collision matrix $\mathbf{S}$ is invertible, and the inverse matrix is given as

$$
\mathbf{S}^{-1}=\left[\begin{array}{ccccccccc}
s_{0}^{-1} & 0 & 0 & 0 & 0 & 0 & 0 & 0 & 0 \\
0 & s_{e}^{-1} & k\left(s_{e}^{-1}-\frac{1}{2}\right) & 0 & h \hat{u}_{x}\left(s_{e}^{-1}-\frac{1}{2}\right) & 0 & h \hat{u}_{y}\left(s_{e}^{-1}-\frac{1}{2}\right) & 0 & 0 \\
0 & 0 & s_{\varepsilon}^{-1} & 0 & 0 & 0 & 0 & 0 & 0 \\
0 & 0 & 0 & s_{j}^{-1} & 0 & 0 & 0 & 0 & 0 \\
0 & 0 & 0 & 0 & s_{q}^{-1} & 0 & 0 & 0 & 0 \\
0 & 0 & 0 & 0 & 0 & s_{j}^{-1} & 0 & 0 & 0 \\
0 & 0 & 0 & 0 & 0 & 0 & s_{q}^{-1} & 0 & 0 \\
0 & 0 & 0 & 0 & 2 b \hat{u}_{x}\left(s_{p}^{-1}-\frac{1}{2}\right) & 0 & -2 b \hat{u}_{y}\left(s_{p}^{-1}-\frac{1}{2}\right) & s_{p}^{-1} & 0 \\
0 & 0 & 0 & 0 & b \hat{u}_{y}\left(s_{p}^{-1}-\frac{1}{2}\right) & 0 & b \hat{u}_{x}\left(s_{p}^{-1}-\frac{1}{2}\right) & 0 & s_{p}^{-1}
\end{array}\right] .
$$

Similar to the second-order analysis, the equations for $m_{0}, m_{3}$, and $m_{5}$ are extracted from Eq. (45) to deduce the macroscopic conservation equation. From the $\varepsilon^{0}$-order equation [i.e., Eq. (45a)], we have

$$
\varepsilon^{0}:\left\{\begin{array}{l}
m_{0}^{(0)}=m_{0}^{\mathrm{eq}} \\
m_{3}^{(0)}=m_{3}^{\mathrm{eq}} \\
m_{5}^{(0)}=m_{5}^{\mathrm{eq}}
\end{array}\right.
$$

which indicate

$$
\begin{cases}m_{0}^{(1)}+\frac{\delta_{t}}{2} F_{m, 0}^{(1)}=0, & m_{0}^{(n)}=0(\forall n \geq 2), \\ m_{3}^{(1)}+\frac{\delta_{t}}{2} F_{m, 3}^{(1)}=0, & m_{3}^{(n)}=0(\forall n \geq 2), \\ m_{5}^{(1)}+\frac{\delta_{t}}{2} F_{m, 5}^{(1)}=0, & m_{5}^{(n)}=0(\forall n \geq 2),\end{cases}
$$

by considering the definition equations for $\rho$ and $\rho \mathbf{u}$. The equations for conserved moments in the $\varepsilon^{1}$-order equation [i.e., Eq. (45b)] can be simplified as

$$
\varepsilon^{1}:\left\{\begin{array}{l}
\partial_{t 1} \rho=0 \\
\partial_{t 1}\left(\rho u_{x}\right)=-\partial_{x 1} p_{\mathrm{LBE}}+F_{x}^{(1)} \\
\partial_{t 1}\left(\rho u_{y}\right)=-\partial_{y 1} p_{\mathrm{LBE}}+F_{y}^{(1)}
\end{array}\right.
$$


where the recovered EOS $p_{\mathrm{LBE}}=c_{s}^{2}\left[\left(2+\alpha_{1}\right) \rho+\beta_{1} \eta\right]$ and the lattice sound speed $c_{s}=c / \sqrt{3}$. Similarly, the equations for conserved moments in the $\varepsilon^{2}$-order equation [i.e., Eq. (45c)] can be simplified as

$$
\varepsilon^{2}:\left\{\begin{array}{l}
\partial_{t 2} \rho=0, \\
\partial_{t 2}\left(\rho u_{x}\right)=0, \\
\partial_{t 2}\left(\rho u_{y}\right)=0 .
\end{array}\right.
$$

After some lengthy algebra, the equations for conserved moments in the $\varepsilon^{3}$-order equation [i.e., Eq. (45d)] can be finally simplified as

$$
\varepsilon^{3}:\left\{\begin{aligned}
& \partial_{t 3} \rho=0, \\
& \partial_{t 3}\left(\rho u_{x}\right)=\frac{1}{12} \delta_{x}^{2} \nabla_{1} \cdot \nabla_{1} F_{x}^{(1)}-\frac{1}{24} c^{2}\left[2 \nabla_{1} \cdot\left(\tilde{\mathbf{F}}^{(1)} \tilde{F}_{x}^{(1)}\right)+\partial_{x 1}\left(\tilde{\mathbf{F}}^{(1)} \cdot \tilde{\mathbf{F}}^{(1)}\right)\right] \\
&-\frac{1}{6} \delta_{x}^{2}\left[(k+1) \tau_{e} \tau_{q}-\tau_{p} \tau_{q}\right] \nabla_{1} \cdot \nabla_{1} \partial_{x 1} \bar{p}-\frac{1}{12} \delta_{x}^{2}\left(12 \tau_{p} \tau_{q}-1\right) \partial_{y 1}^{2} \partial_{x 1} \bar{p}, \\
& \partial_{t 3}\left(\rho u_{y}\right)=\frac{1}{12} \delta_{x}^{2} \nabla_{1} \cdot \nabla_{1} F_{y}^{(1)}-\frac{1}{24} c^{2}\left[2 \nabla_{1} \cdot\left(\tilde{\mathbf{F}}^{(1)} \tilde{F}_{y}^{(1)}\right)+\partial_{y 1}\left(\tilde{\mathbf{F}}^{(1)} \cdot \tilde{\mathbf{F}}^{(1)}\right)\right] \\
&-\frac{1}{6} \delta_{x}^{2}\left[(k+1) \tau_{e} \tau_{q}-\tau_{p} \tau_{q}\right] \nabla_{1} \cdot \nabla_{1} \partial_{y 1} \bar{p}-\frac{1}{12} \delta_{x}^{2}\left(12 \tau_{p} \tau_{q}-1\right) \partial_{x 1}^{2} \partial_{y 1} \bar{p},
\end{aligned}\right.
$$

where $\tau_{e, p, q}=s_{e, p, q}^{-1}-1 / 2$ and $\bar{p}=c_{s}^{2}\left[\left(3 \alpha_{1}+\alpha_{2}+2\right) \rho+\left(3 \beta_{1}+\beta_{2}\right) \eta\right]$. With the ordinary choices $\alpha_{1}=-1$ and $\beta_{1}=1$, $\bar{p}$ is then given as $\bar{p}=\left(3+\beta_{2}\right) c_{s}^{2} \eta$. Combining the $\varepsilon^{1}-, \varepsilon^{2}$-, and $\varepsilon^{3}$-order equations [i.e., Eqs. (48), (49), and (50)], the macroscopic equation in steady and stationary situation at the $\varepsilon^{3}$-order can be recovered as follows

$$
\left\{\begin{array}{l}
\partial_{t} \rho=0 \\
\partial_{t}(\rho \mathbf{u})=-\nabla p_{\mathrm{LBE}}+\mathbf{F}+\mathbf{R}_{\text {iso }}+\mathbf{R}_{\text {add }}+\overline{\mathbf{R}}_{\text {iso }}+\overline{\mathbf{R}}_{\text {aniso }},
\end{array}\right.
$$

where $\mathbf{R}_{\text {iso }}$ denotes the isotropic term caused by the discrete lattice effect on the force term, $\mathbf{R}_{\text {add }}$ denotes the additional term introduced by the term $\mathbf{S Q}_{m}$ in LB equation, $\overline{\mathbf{R}}_{\text {iso }}$ and $\overline{\mathbf{R}}_{\text {aniso }}$ denote the isotropic and anisotropic terms, respectively, caused by $\eta$ that is introduced to achieve self-tuning EOS, and these $\varepsilon^{3}$-order terms are expressed as

$$
\begin{array}{ll}
\mathbf{R}_{\text {iso }}=\frac{1}{12} \delta_{x}^{2} \nabla \cdot \nabla \mathbf{F}, & \mathbf{R}_{\text {add }}=-\frac{1}{24} c^{2} \nabla \cdot[2 \tilde{\mathbf{F}} \tilde{\mathbf{F}}+(\tilde{\mathbf{F}} \cdot \tilde{\mathbf{F}}) \mathbf{I}], \\
\overline{\mathbf{R}}_{\text {iso }}=-\frac{1}{6} \delta_{x}^{2}\left[(k+1) \tau_{e} \tau_{q}-\tau_{p} \tau_{q}\right] \nabla \cdot \nabla \nabla \bar{p}, & \overline{\mathbf{R}}_{\text {aniso }}=-\frac{1}{12} \delta_{x}^{2}\left(12 \tau_{p} \tau_{q}-1\right)\left(\partial_{y}^{2} \partial_{x} \bar{p}, \partial_{x}^{2} \partial_{y} \bar{p}\right)^{\mathrm{T}} .
\end{array}
$$

For multiphase flows, the anisotropic term $\overline{\mathbf{R}}_{\text {aniso }}$ must be eliminated based on our previous analysis [4], and the isotropic term $\overline{\mathbf{R}}_{\text {iso }}$ should also be eliminated since it is related to the relaxation parameters. For these purposes, we can set

$$
\tau_{p} \tau_{q}=(k+1) \tau_{e} \tau_{q}=\frac{1}{12}
$$

\section{A. Pressure tensor}

With the consideration of the $\varepsilon^{3}$-order terms $\mathbf{R}_{\text {iso }}$ and $\mathbf{R}_{\text {add }}$, the pressure tensor $\mathbf{P}$ recovered by the present LB model is defined as

$$
\nabla \cdot \mathbf{P}=\nabla p_{\mathrm{LBE}}-\mathbf{F}-\mathbf{R}_{\text {iso }}-\mathbf{R}_{\text {add }} .
$$

Performing Taylor series expansion of $\rho\left(\mathbf{x}+\mathbf{e}_{i} \delta_{t}\right)$ centered at $\mathbf{x}$, the interaction force $\mathbf{F}$ can be expressed as

$$
\mathbf{F}=G^{2} \delta_{x}^{2} \rho \nabla \rho+\frac{G^{2} \delta_{x}^{4}}{6} \rho \nabla \nabla \cdot \nabla \rho+O\left(\partial_{x, y}^{5}\right) .
$$

Therefore, $\mathbf{F}+\mathbf{R}_{\text {iso }}$ in Eq. (54) can be simplified as

$$
\begin{aligned}
\mathbf{F}+\mathbf{R}_{\text {iso }}= & G^{2} \delta_{x}^{2} \rho \nabla \rho+\frac{G^{2} \delta_{x}^{4}}{6} \rho \nabla \nabla \cdot \nabla \rho+\frac{G^{2} \delta_{x}^{4}}{12} \nabla \cdot \nabla(\rho \nabla \rho)+O\left(\partial_{x, y}^{5}\right) \\
= & \frac{G^{2} \delta_{x}^{2}}{2} \nabla \cdot\left(\rho^{2} \mathbf{I}\right)+\frac{G^{2} \delta_{x}^{4}}{6} \nabla \cdot\left[a_{1} \nabla \rho \nabla \rho+a_{2} \rho \nabla \nabla \rho+\left(a_{3} \nabla \rho \cdot \nabla \rho+a_{4} \rho \nabla \cdot \nabla \rho\right) \mathbf{I}\right] \\
& +\frac{G^{2} \delta_{x}^{4}}{12} \nabla \cdot\left[b_{1} \nabla \rho \nabla \rho+b_{1} \rho \nabla \nabla \rho+\left(b_{2} \nabla \rho \cdot \nabla \rho+b_{2} \rho \nabla \cdot \nabla \rho\right) \mathbf{I}\right]+O\left(\partial_{x, y}^{5}\right),
\end{aligned}
$$


where $a_{1,2,3,4}$ and $b_{1,2}$ are free parameters that satisfy

$$
a_{1}+a_{2}+2 a_{3}=0, \quad a_{1}+a_{4}=0, \quad a_{2}+a_{4}=1 ; \quad b_{1}+b_{2}=1 .
$$

Although these free parameters cannot be completely determined, they make no difference to the thermodynamic properties predicted by the pressure tensor [4]. Therefore, we choose $2 a_{2}+b_{1}=0$ as a supplementary constraint for the sake of simplicity. Then, Eq. (56) can be further simplified as

$$
\mathbf{F}+\mathbf{R}_{\text {iso }}=\frac{G^{2} \delta_{x}^{2}}{2} \nabla \cdot\left(\rho^{2} \mathbf{I}\right)+\frac{G^{2} \delta_{x}^{4}}{12} \nabla \cdot[-2 \nabla \rho \nabla \rho+(2 \nabla \rho \cdot \nabla \rho+3 \rho \nabla \cdot \nabla \rho) \mathbf{I}]+O\left(\partial_{x, y}^{5}\right) .
$$

By utilizing Eq. (55), $\mathbf{R}_{\text {add }}$ can be simplified as

$$
\mathbf{R}_{\text {add }}=-\frac{G^{2} \delta_{x}^{4}}{24} \nabla \cdot[2 \nabla \rho \nabla \rho+(\nabla \rho \cdot \nabla \rho) \mathbf{I}]+O\left(\partial_{x, y}^{5}\right) .
$$

Based on Eqs. (58) and (59), the pressure tensor defined by Eq. (54) can be finally derived as follows

$$
\begin{aligned}
\mathbf{P} & =\left(p_{\mathrm{LBE}}-\frac{G^{2} \delta_{x}^{2}}{2} \rho^{2}-\frac{G^{2} \delta_{x}^{4}}{4} \rho \nabla \cdot \nabla \rho-\frac{G^{2} \delta_{x}^{4}}{8} \nabla \rho \cdot \nabla \rho\right) \mathbf{I}+\frac{G^{2} \delta_{x}^{4}}{4} \nabla \rho \nabla \rho+O\left(\partial_{x, y}^{4}\right) \\
& =\left(p_{\mathrm{EOS}}-\kappa \rho \nabla \cdot \nabla \rho-\frac{\kappa}{2} \nabla \rho \cdot \nabla \rho\right) \mathbf{I}+\kappa \nabla \rho \nabla \rho+O\left(\partial_{x, y}^{4}\right),
\end{aligned}
$$

where $G^{2} \delta_{x}^{4} / 4$ is denoted by $\kappa$, and $p_{\mathrm{LBE}}-G^{2} \delta_{x}^{2} \rho^{2} / 2$ is, namely, the EOS $p_{\mathrm{EOS}}$.

\section{IMPLEMENTATION}

At first glance, the present collision process [Eq. (1)] seems difficult to implement. However, in real applications, it can be executed in the following sequence

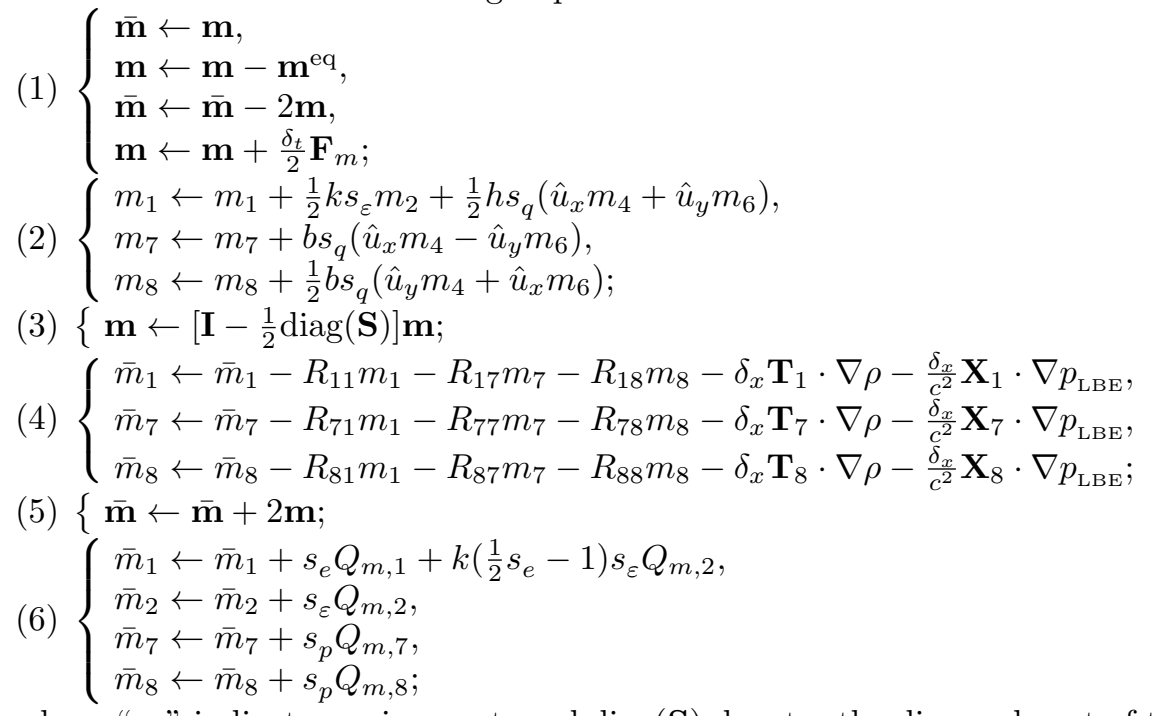

where " $\leftarrow$ " indicates assignment, and $\operatorname{diag}(\mathbf{S})$ denotes the diagonal part of the present collision matrix $\mathbf{S}$. Here, steps (1), (3), and (5) are the same as those for the classical multiple-relaxation-time (MRT) collision process, step (2) corresponds to the improvement of the collision matrix, step (4) corresponds to the elimination of the cubic terms of velocity, and step (6) corresponds to the introduction of the $\varepsilon^{3}$-order additional term. In step (4), $\nabla \rho$ and $\nabla p_{\mathrm{LBE}}$ can be locally evaluated resorting to the interaction force as follows

$$
\begin{aligned}
& \nabla \rho=\frac{1}{\delta_{x}^{2}} \sum_{i} \omega\left(\left|\mathbf{e}_{i} \delta_{t}\right|^{2}\right) \rho\left(\mathbf{x}+\mathbf{e}_{i} \delta_{t}\right) \mathbf{e}_{i} \delta_{t}=\frac{\mathbf{F}}{G^{2} \delta_{x}^{2} \rho}, \\
& \nabla p_{\mathrm{LBE}}=\nabla\left(p_{\mathrm{EOS}}+\frac{G^{2} \delta_{x}^{2}}{2} \rho^{2}\right)=\left(\frac{d p_{\mathrm{EOS}}}{d \rho}+G^{2} \delta_{x}^{2} \rho\right) \frac{\mathbf{F}}{G^{2} \delta_{x}^{2} \rho} .
\end{aligned}
$$

From the above discussion, it can be seen that the present collision process is actually easy to implement, and all the involved computations can be locally performed except for the simple interaction force. Therefore, compared with 
the pseudopotential LB model, known as the simplest multiphase LB model, no additional computational complexity is introduced in the present LB model. Based on our numerical test, the computational cost of the present model is only 1.178 times as much as that of a pseudopotential MRT LB model by Li et al. [5].

\section{VALIDATIONS}

In the following numerical validations, the basic simulation parameters are set as $\varpi=1 / 6, a=1, b=4, R=1$, and $\delta_{x}=1$. Firstly, one-dimensional flat interface is simulated to validate the relation $W \propto K_{\text {INT }}$. Here, the interface thickness is measured from $\rho=0.95 \rho_{g}+0.05 \rho_{l}$ to $0.05 \rho_{g}+0.95 \rho_{l}$, with $\rho_{g}$ and $\rho_{l}$ denoting the coexistence gas and liquid densities, respectively. Fig. 1 shows the variation of $W$ with $K_{\text {INT }}$ for the reduced temperature $T_{r}=T / T_{c}$ being 0.9 , $0.8,0.7,0.6$, and 0.5 , and the scaling factor $K_{\text {EOS }}$ being 1 . Good proportionality between $W$ and $K_{\text {INT }}$ can be clearly seen, and the proportionality constants (i.e., the slopes of the dashed lines) are 6.3682, 4.3490, 3.4403, 2.8787, and 2.5178 for $T_{r}=0.9,0.8,0.7,0.6$, and 0.5 , respectively, which agree very well with the corresponding analytical results $\left(6.3711,4.3574,3.4423,2.8875\right.$, and 2.5052 for $T_{r}=0.9,0.8,0.7,0.6$, and 0.5 , respectively) predicted by the pressure tensor. Note that when $T_{r}$ is relatively small (such as $T_{r}=0.5$ ), $K_{\mathrm{INT}}$ should be increased to widen the interfacial region for numerical stability in the simulation. Fig. 2 shows the density profile across interface for $K_{\mathrm{EOS}}=10^{-2}$, $10^{-1}, 10^{0}, 10^{1}$, and $10^{2}$, and $T_{r}=0.8$. Here, the interface thickness is fixed at $W=10$ (i.e., $\left.K_{\text {INT }}=2.2949\right)$. It can be seen that the density profiles for different $K_{\mathrm{EOS}}$ are identical, which indicates that both the coexistence densities and interface thickness are not affected by $K_{\mathrm{Eos}}$. Moreover, the density profile obtained by simulation is in good agreement with the analytical one predicted by the pressure tensor, which further validates the present LB model for multiphase flows.

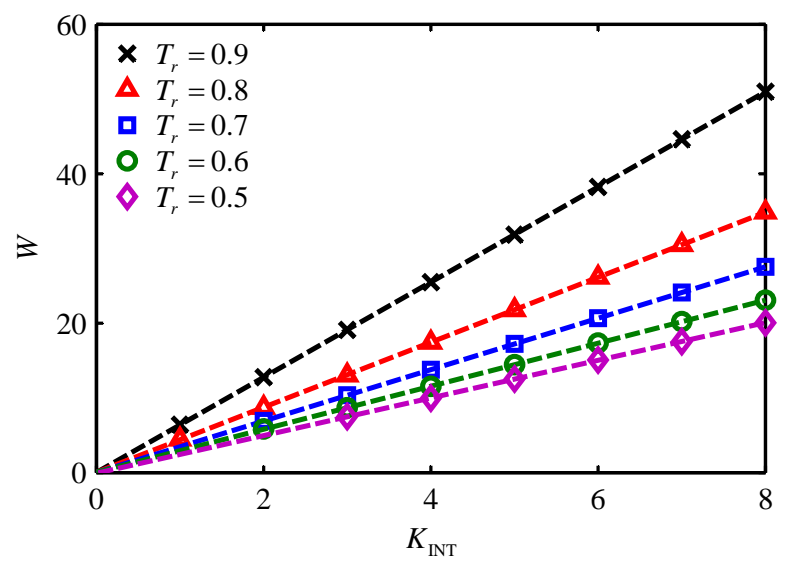

FIG. 1. Variation of the interface thickness $W$ with the scaling factor $K_{\text {INT }}$. The reduced temperature $T_{r}$ is chosen as 0.9 , $0.8,0.7,0.6$, and 0.5 , respectively, and the scaling factor $K_{\mathrm{EOS}}$ is fixed at 1 . The dashed lines, obtained by linear fitting in a least-squares sense, are given as $6.3682 K_{\mathrm{INT}}+0.0156,4.3490 K_{\mathrm{INT}}+0.0392,3.4403 K_{\mathrm{INT}}+0.0218,2.8787 K_{\mathrm{INT}}+0.0527$, and $2.5178 K_{\mathrm{INT}}-0.0829$ for $T_{r}=0.9,0.8,0.7,0.6$, and 0.5 , respectively.

Numerical simulation of two-dimensional circular droplet is then carried out to validate the relation $\sigma \propto K_{\mathrm{EOS}} K_{\text {INT }}$. Here, the surface tension is computed via Laplace's law, i.e., $\sigma=\left(p_{\text {in }}-p_{\text {out }}\right) D / 2$ with $p_{\text {in }}$ and $p_{\text {out }}$ denoting the pressure inside and outside the droplet, respectively, and $D$ being the droplet diameter measured at $\rho=\left(\rho_{g}+\rho_{l}\right) / 2$. Fig. 3 shows the variation of $\sigma$ with $K_{\mathrm{INT}}$ for $T_{r}=0.9,0.8,0.7,0.6$, and 0.5 , and the scaling factor $K_{\mathrm{EOS}}=1$. As seen, $\sigma$ is indeed proportional to $K_{\mathrm{INT}}$. The proportionality constants are $0.003157,0.009115,0.01716,0.02725$, and 0.03979 for $T_{r}=0.9,0.8,0.7,0.6$, and 0.5 , respectively, which are in good agreement with the corresponding analytical results (0.003146, 0.009081, 0.01710, 0.02716, and 0.03954 for $T_{r}=0.9,0.8,0.7,0.6$, and 0.5 , respectively) predicted by the pressure tensor. Compared with the simulation of flat interface, $K_{\text {INT }}$ required for numerical stability significantly increases when $T_{r}=0.5$. Fig. 4 shows the variation of $\sigma$ with $K_{\mathrm{Eos}}$ for various $T_{r}$. Here, the interface thickness is fixed at $W=20$ for $T_{r} \geq 0.6$ and 30 for $T_{r}=0.5$. The results demonstrate the perfect proportionality between $\sigma$ and $K_{\text {EOS }}$, and also suggest that $\sigma$ can be adjusted in a wide range via $K_{\text {Eos }}$. 


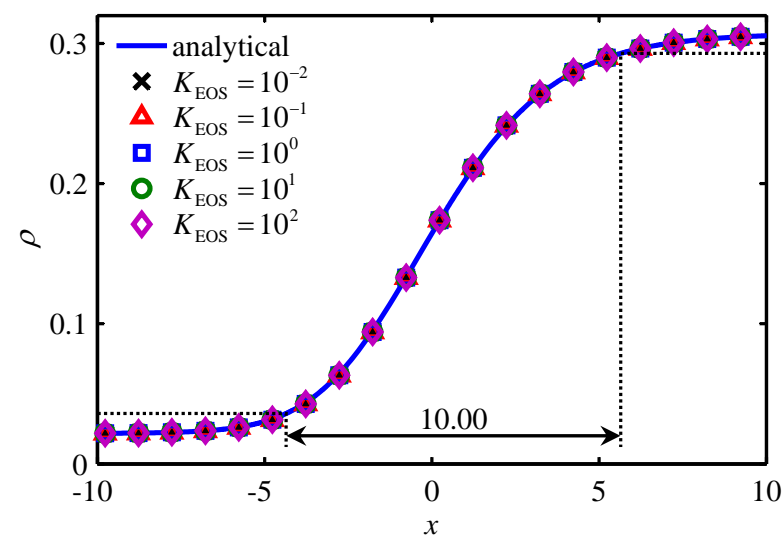

FIG. 2. Density profiles across interface obtained with various $K_{\mathrm{EOS}}$. The reduced temperature $T_{r}$ and interface thickness $W$ are fixed at 0.8 and 10 , respectively. The solid line is the analytical profile predicted by pressure tensor. The dotted lines depict the interfacial region defined from $\rho=0.95 \rho_{g}+0.05 \rho_{l}$ to $0.05 \rho_{g}+0.95 \rho_{l}$.

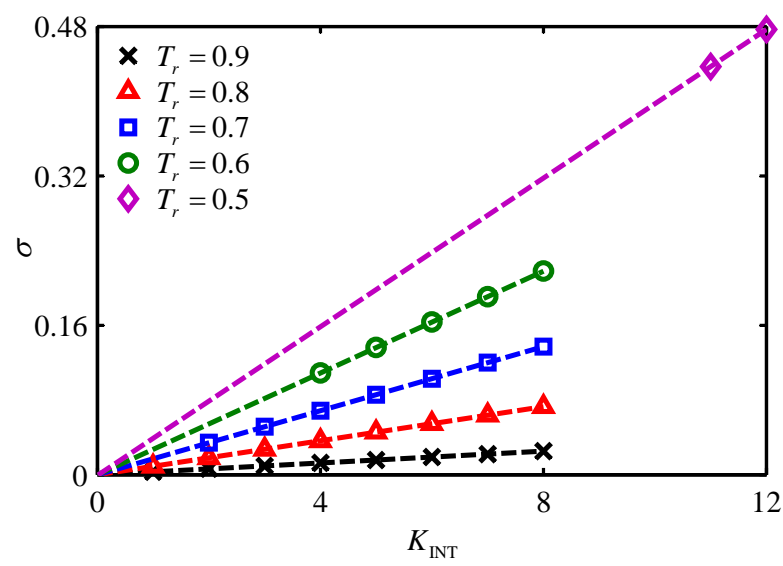

FIG. 3. Variation of the surface tension $\sigma$ with the scaling factor $K_{\text {INT }}$. The reduced temperature $T_{r}$ is chosen as 0.9 , 0.8 , $0.7,0.6$, and 0.5 , respectively, and the scaling factor $K_{\mathrm{EOS}}$ is fixed at 1 . The dashed lines, obtained by linear fitting in a least-squares sense, are given as $0.003157 K_{\mathrm{INT}}-3.060 \times 10^{-5}, 0.009115 K_{\text {INT }}-8.310 \times 10^{-5}, 0.01716 K_{\text {INT }}-8.398 \times 10^{-5}$, $0.02725 K_{\mathrm{INT}}-2.251 \times 10^{-5}$, and $0.03979 K_{\mathrm{INT}}-7.627 \times 10^{-4}$ for $T_{r}=0.9,0.8,0.7,0.6$, and 0.5 , respectively.

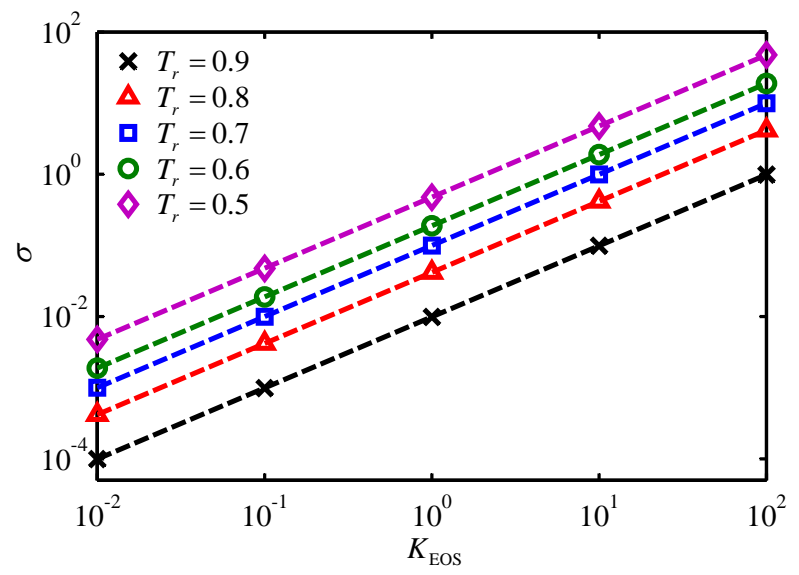

FIG. 4. Variation of the surface tension $\sigma$ with the scaling factor $K_{\mathrm{EOS}}$. The reduced temperature $T_{r}$ is chosen as $0.9,0.8,0.7$, 0.6 , and 0.5, respectively, and the interface thickness $W$ is fixed at 20 for $T_{r} \geq 0.6$ and 30 for $T_{r}=0.5$, respectively. The dashed lines, obtained by linear fitting in a least-squares sense, are given as $0.009879 K_{\mathrm{EOS}}+3.570 \times 10^{-17}, 0.04174 K_{\mathrm{EOS}}+1.428 \times 10^{-16}$, $0.09959 K_{\mathrm{EOS}}+3.427 \times 10^{-16}, 0.1887 K_{\mathrm{EOS}}+6.283 \times 10^{-16}$, and $0.4758 K_{\mathrm{EOS}}+1.059 \times 10^{-7}$ for $T_{r}=0.9,0.8,0.7,0.6$, and 0.5 , respectively. 
[1] Y. H. Qian, D. d'Humières, and P. Lallemand, Europhys. Lett. 17, 479 (1992).

[2] P. Lallemand and L.-S. Luo, Phys. Rev. E 61, 6546 (2000).

[3] S. Chapman and T. G. Cowling, The Mathematical Theory of Non-Uniform Gases, 3rd ed. (Cambridge University Press, Cambridge, 1970).

[4] R. Huang and H. Wu, J. Comput. Phys. 327, 121 (2016).

[5] Q. Li, K. H. Luo, and X. J. Li, Phys. Rev. E 87, 053301 (2013). 